San Jose State University

SJSU ScholarWorks

Master's Theses

Master's Theses and Graduate Research

Summer 2010

\title{
Host-Parasite Ecology and Description of the Louse Fly, Allobosca crassipes, from Madagascar
}

Stanley Edmondson Vaughn

San Jose State University

Follow this and additional works at: https://scholarworks.sjsu.edu/etd_theses

\section{Recommended Citation}

Vaughn, Stanley Edmondson, "Host-Parasite Ecology and Description of the Louse Fly, Allobosca crassipes, from Madagascar" (2010). Master's Theses. 3835.

DOI: https://doi.org/10.31979/etd.anx2-e557

https://scholarworks.sjsu.edu/etd_theses/3835

This Thesis is brought to you for free and open access by the Master's Theses and Graduate Research at SJSU ScholarWorks. It has been accepted for inclusion in Master's Theses by an authorized administrator of SJSU ScholarWorks. For more information, please contact scholarworks@sjsu.edu. 
HOST-PARASITE ECOLOGY AND DESCRIPTION OF THE LOUSE FLY, ALLOBOSCA CRASSIPES, FROM MADAGASCAR

\author{
A Thesis \\ Presented to \\ The Faculty of the Department of Biological Sciences \\ San José State University
}

\author{
In Partial Fulfillment \\ of the Requirements for the Degree \\ Master of Science
}

by

Stanley E. Vaughn

August 2010 
(C) 2010

Stanley E. Vaughn

ALL RIGHTS RESERVED 
The Designated Thesis Committee Approves the Thesis Titled

HOST-PARASITE ECOLOGY AND DESCRIPTION OF THE LOUSE FLY, ALLOBOSCA CRASSIPES, FROM MADAGASCAR

by

Stanley E. Vaughn

APPROVED FOR THE DEPARTMENT OF BIOLOGICAL SCIENCES

SAN JOSÉ STATE UNIVERSITY

August 2010

Dr. Elizabeth McGee

Department of Biological Sciences

Dr. Shannon Bros-Seeman

Department of Biological Sciences

Dr. Paul H. Arnaud, Jr.

California Academy of Sciences 


\begin{abstract}
HOST-PARASITE ECOLOGY AND DESCRIPTION OF THE LOUSE FLY, ALLOBOSCA CRASSIPES, FROM MADAGASCAR
\end{abstract}

\author{
by Stanley E. Vaughn
}

The louse fly, Allobosca crassipes (Diptera: Hippoboscidae), is an obligate ectoparasite exclusive to lemurs in Madagascar. Research in Ranomafana National Park (RNP) in southeastern Madagascar has resulted in the identification of a new association of this fly with the black and white ruffed lemur, Varecia variegata variegata, and a confirmed association with Milne-Edwards’ sifaka, Propithecus edwardsi. Previous descriptions of $A$. crassipes were based on only a few specimens, and the type specimen is presumed destroyed. The specimens of A. crassipes collected by SJSU in 2000-2001 provide the largest collection available for the purpose of describing this species.

Habitat disturbance is a major threat to the biodiversity of RNP and is predicted to affect parasite-host interactions. Disturbance has changed the behavior of the lemur hosts, which has indirectly affected the parasites. A. crassipes is found on several lemur species that rarely interact, but its low host specificity is an enigma since it is wingless as an adult. Disturbance may, however, indirectly contribute to low host specificity by forcing proximity and increasing interaction between host species. Further evidence of disturbance is seen in the isotopic composition of $A$. crassipes. A. crassipes-parasitizing lemurs inhabiting disturbed forests are significantly nitrogen depleted (as are their hosts) vs. those inhabiting undisturbed forests. These data further validate stable isotope ecology as a means of identifying ecosystem-wide habitat disturbance in rainforests. 


\section{ACKNOWLEDGEMENTS}

Many people have been involved in this project over the last several years. I thank the Madagascar Ministry of the Interior and the Madagascar National Parks for authorization to conduct fieldwork in RNP. I am indebted to the following individuals for their invaluable advice and/or logistical support: Dr. Elizabeth Erhart (Southwest Texas State University), Dr. Patricia Wright (SUNY Stony Brook), Dr. Summer Arrigo-Nelson (California University), Heritiana Ranarivelo (California Academy of Sciences), Dr. Steve King (SUNY Stony Brook), Drs. Deborah Overdorff and Thomas Mutschler (University of Texas), Francois Ratalata, Madame (Jeanne) Aimee, Paul Rasabo, Richard Randriamamjinona and his team of darters, Dr. Benjamin Andriamihaja (MICET, Antananarivo). Dr. Bill Murray and Mr. Larry Young (San Jose State University) provided veterinary support and advice. Special thanks to Virginia Kirsch, Donna Hurth, Ursula Kress, Ellen Corts, Craig Cook (SIRFIR), Keve Ribardo, and Dr. Neil Evenhuse (Bishop Museum). The Michael Lee Environmental Foundation generously provided financial support. I greatly appreciate the advice of my committee members Dr. Shannon Bros-Seeman (SJSU), Dr. Paul H. Arnaud, Jr. (California Academy of Sciences), and my thesis advisor and friend Dr. Elizabeth McGee (SJSU). My sincerest thanks to the late .J. Gordon “Doc”Edwards, and to Dr. Ron Stecker, whose passion for entomology ignited my own and to whom I dedicate this thesis. Ron, you changed my life.

Blut ist ein ganz besondrer Saft

Blood is a special juice

- Goethe’s Faust Part I 


\section{TABLE OF CONTENTS}

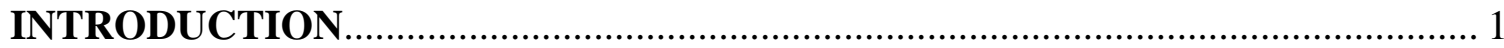

CHAPTER I: Description of Study Sites in Ranomafana National Park ................ 3

INTRODUCTION ...................................................................................... 3

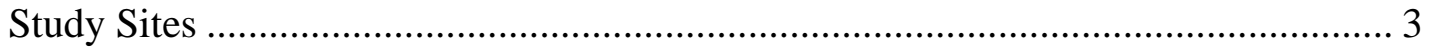

Scope of Research at RNP from 2000-2001 ....................................................... 5

CHAPTER II: Description of Allobosca crassipes (Hippoboscidae: Diptera)............ 7

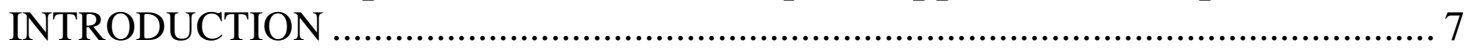

MATERIALS AND METHODS

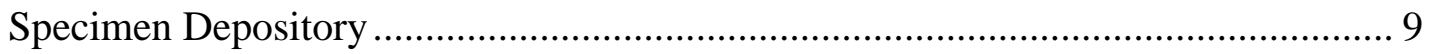

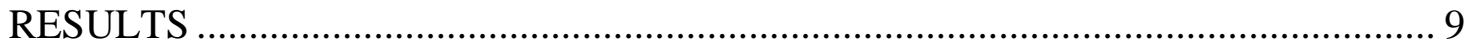

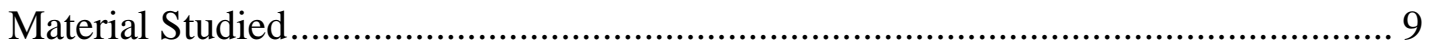

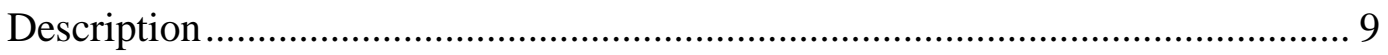

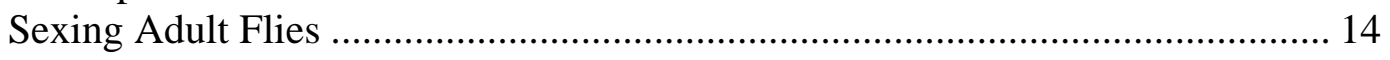

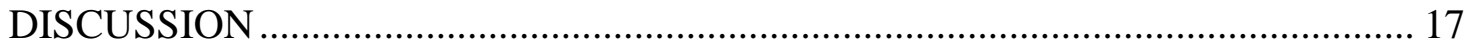

CHAPTER III: Association of Allobosca crassipes (Diptera: Hippoboscidae) with the Black and White Ruffed Lemur (Varecia variegata variegata) and Milne-

Edwards' Sifaka (Propithecus edwardsi) in Southeastern Madagascar .................... 20

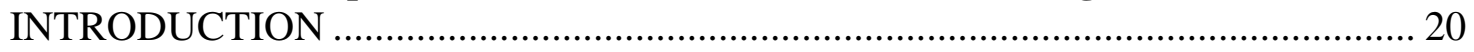

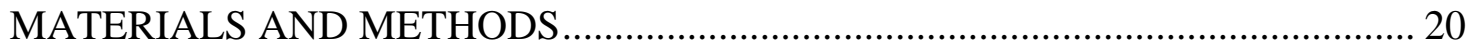

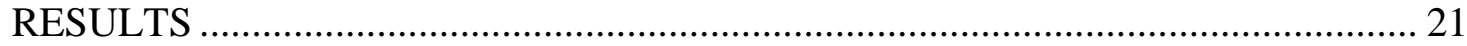

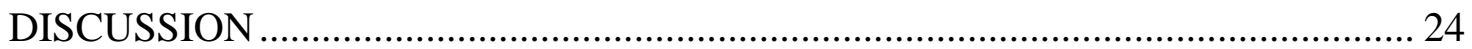

CHAPTER IV: Effect of Habitat Disturbance on Stable Isotope Composition of

Allobosca crassipes (Diptera: Hippoboscidae) from Southeastern Madagascar ....... 27

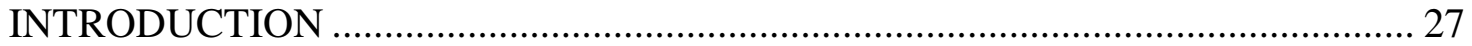

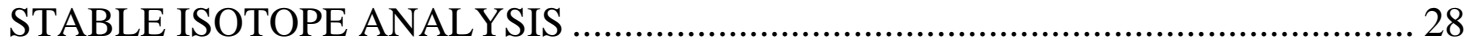

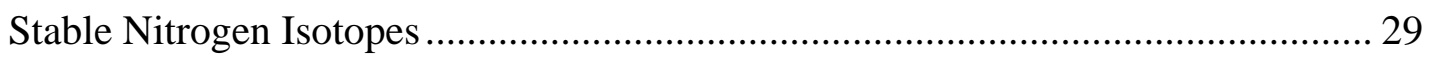

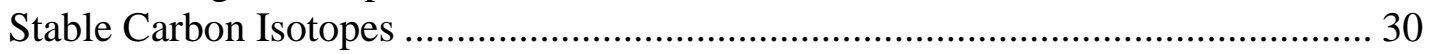

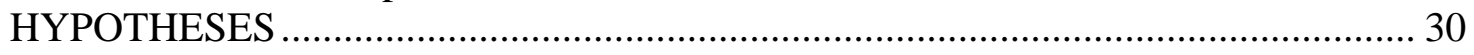

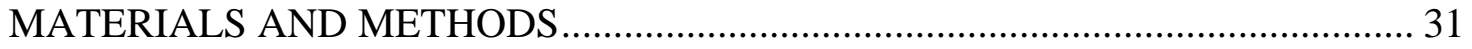

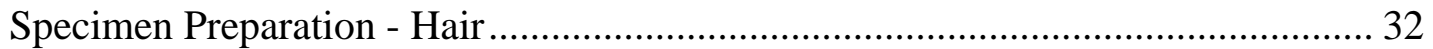

Specimen Preparation - Parasites ....................................................................... 32

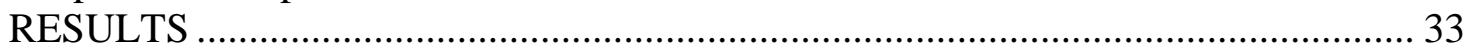

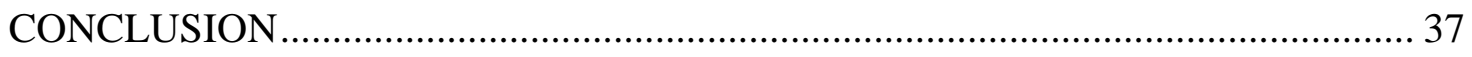

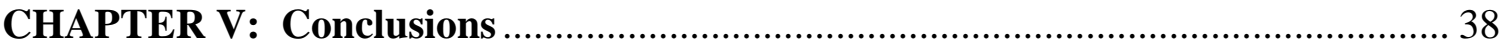

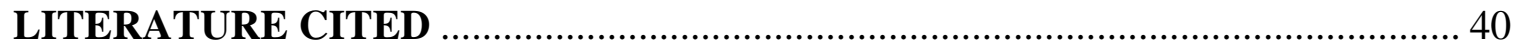




\section{LIST OF FIGURES}

Figure 1 Map of Ranomafana National Park, Madagascar .......................4

Figure 2 Allobosca crassipes, male, dorsal view ..................................13

Figure $3 \quad$ Allobosca crassipes, wing.....................................................15

Figure $4 \quad$ Allobosca crassipes, abdomen ..............................................16

Figure 5 Biogeochemical Profile of Propithecus edwardsi .......................34

Figure $6 \quad$ Biogeochemical Profile of Allobosca crassipes with

Propithecus edwardsi...........................................................36 


\section{LIST OF TABLES}

Table 1 Physical and Botanical Characteristics of Sites in Ranomafana National Park ..........................................................................6

Table 2 List of Flies by Individual Lemur Hosts ......................................10

Table 3 Average Terrestrial Abundance for Carbon and Nitrogen............29

Table $4 \quad$ Count of A. crassipes by Locality ..................................................32

Table $5 \quad$ Stable Isotope Values for A. crassipes...........................................35 


\section{INTRODUCTION}

Madagascar is the fourth largest island in the world and lies $400 \mathrm{~km}$ to the east coast of Mozambique, Africa in the Indian Ocean. This geographic isolation is the result of separation from Africa some 165 million years ago (Rabinowitz 1983). It has been isolated in its present position for over 88 million years (Krause 2003) The effects of this geographic isolation are evident from the 9,703 plant, 771 vertebrate and 2,305 macro invertebrate species that have been described as endemic to the island (Myers et al. 2000; Goodman \& Benstead 2003). When considering the numbers of endemic flora and fauna, the rate of endemism and habitat loss, Madagascar ranks the "hottest" of the world's biodiversity hotspots (Myers et al. 2000). The unique primate fauna of Madagascar comprises more than fifty distinct taxa (Wright 1992), including Milne Edwards' sifaka (Propithecus edwardsi) and the Black and White Ruffed lemur (Varecia variegata variegata). Following her discovery in the 1980s of a rare lemur species, the Golden Bamboo Lemur (Hapalemur aureus), Dr. Patricia Wright convinced the government of Madagascar to establish a national park (Ranomafana National Park, RNP) to protect the flora and fauna of this region. Dr. Wright also established a research station and encouraged cross disciplinary researchers from all over the world, including SJSU, to work in RNP. The work presented here on association of the parasitic Louse-fly Allobosca crassipes (Speiser) with P. edwardsi and V. variegata variegata, and the effects of habitat disturbance on the ecological relationship of these species is the result of this invitation and the subject of this study. 
Chapter I describes the physical and biological characteristics of the three study sites in Ranomafana National Park, Madagascar. Chapter II addresses taxonomic inaccuracies and inconsistencies that historically have been problematic in the identification of Allobosca crassipes. Chapter III, which was published in Pan-Pacific Entomologist in 2009, describes the parasite-host association of A. crassipes with the Black and White Ruffed lemur, (Varecia variegata variegata), and Milne Edwards' sifaka (Propithecus edwardsi), and finally, Chapter IV discusses the effect of habitat disturbance on the stable isotope composition of A. crassipes and one of its host species, P. edwardsi. The thesis concludes in Chapter V with a summary of this research. 


\section{CHAPTER I: Description of Study Sites in Ranomafana National Park}

\section{INTRODUCTION}

Ranomafana National Park (RNP) is located between $47^{\circ} 18^{\prime}-47^{\circ} 37^{\prime}$ East and $21^{\circ} 02^{\prime}-21^{\circ} 25^{\prime}$ South on the east-facing escarpment of the central high plateau in southeastern Madagascar (Figure 1). The park comprises 43,000 ha and ranges in elevation from 500-1500m (Dew \& Wright 1998; Balko \& Underwood 2005). Although the park is surrounded by a buffer zone of mostly secondary forest, much of this border is agricultural land that is used by several villages. The use of this land by these villages includes the harvesting of timber for construction and the collection of medicinal plants (Wright 1992). In 2000-2001 SJSU conducted research in RNP at three locations:

Talatakely, Vatoharanana and Valohoaka. These three sites vary in extent of anthropogenic impact and provide an opportunity to compare the impact of selective commercial logging on this rainforest ecosystem.

\section{Study Sites}

Talatakely, a small village from 1935 to 1947 , comprises approximately $3 \mathrm{~km}^{2}$ and was intensely selectively logged from 1986-90 (Wright 1997). Talatakely is considered the most anthropogenically disturbed habitat within RNP, and is a mixed primary/secondary forest. The location of the first research station in Talatekely from 1986-2003 also contributed to pressure on the local environment. Vatoharanana and Valohoaka, in contrast, are primary forests further to the South. Vatoharanana, which is $6 \mathrm{~km}$ southeast of Talatakely, comprises approximately $8 \mathrm{~km}^{2}$ and was moderately 


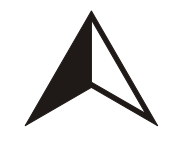

- low altitude sites

- medium altitude sites

* high altitude sites

Scale: $1 / 350,000$
Limit of the buffer zone

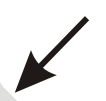

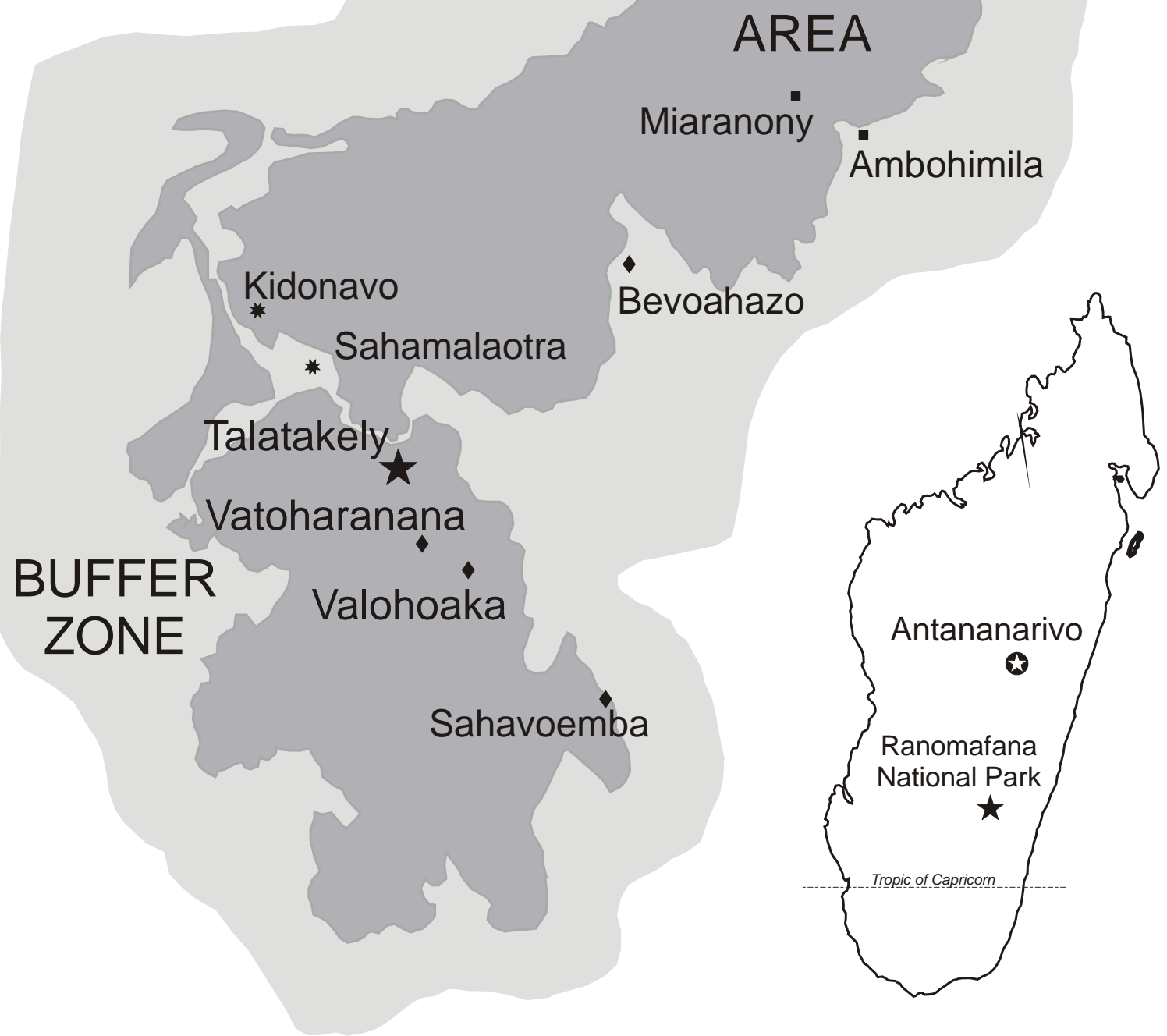

Figure 1: Ranomafana National Park, Madagascar (after Wright 1992, 1997) 
selectively logged from 1987-88. Valohoaka is approximately $8.5 \mathrm{~km}$ southeast of Talatakely and consists of $9 \mathrm{~km}^{2}$. A moratorium on logging in 1988 prohibited commercial operations in Valohoaka thereby ensuring that the forest remained relatively intact (Balko \& Underwood 2005). Physical and botanical parameters of the three sites are given in Table 1.

\section{Scope of Research at RNP from 2000-2001}

The field research conducted by investigators present in RNP during the period of SJSU's work in 2000-2001 was quite diverse. Because the work of several researchers required the darting and sedation of lemurs, it was important to synchronize and coordinate our efforts to ensure that as much data could be collected by different research teams during a single procedure. For example, Patricia Wright's team collected morphometric data on P. diadema as part of a long-term study on the demography of this species (Pochron et al. 2004). Major et al. (2006) collected skin samples for genetic analysis as part of a study on the systematic status of Propithecus. McGee et al. (2001, 2002) collected hair samples to study the biogeochemistry of P. edwardsi. Other researchers fitted individual lemurs with radio tracking collars to monitor the movements

of individuals and groups. For example, Arrigo-Nelson (2006) followed P. edwardsi to study the feeding behavior of groups inhabiting disturbed and undisturbed habitats. The collection of ectoparasites for this project from these lemurs was part of this coordinated effort, and the results of this research are described in the following chapters. 
Table 1: Physical and Botanical Characteristics of Sites in Ranomafana National Park

\begin{tabular}{|c|c|c|c|}
\hline & Talatakely & Vatoharanana & Valohoaka \\
\hline Altitude (m) & 900 & 1125* & 1200 \\
\hline Mean tree height $(\mathrm{m}) \dagger$ & 6.7 & 9.1 & not available \\
\hline Mean tree height $(\mathrm{m}) \S$ & 13.22 & 15.07 & 14.82 \\
\hline Mean tree height $(\mathrm{m}) \ddagger$ & 10.05 & not available & 11.76 \\
\hline Mean tree DBH $(\mathrm{cm}) \dagger$ & 21.5 & 23.3 & not available \\
\hline Mean tree $\mathrm{DBH}(\mathrm{cm}) \ddagger$ & 11.83 & not available & 13.31 \\
\hline Mean tree $\mathrm{DBH}(\mathrm{cm}) \S$ & 19.76 & 31.20 & 29.38 \\
\hline Mean crown volume $\left(\mathrm{m}^{3}\right) \diamond$ & 198.52 & 238.73 & 259.47 \\
\hline Mean crown volume $\left(\mathrm{m}^{3}\right) \ddagger$ & 42.56 & not available & 72.41 \\
\hline Forest type & Mixed primary/secondary & Primary & Primary \\
\hline $\begin{array}{l}\text { History of anthropogenic } \\
\text { impact } \\
\text { to }\end{array}$ & $\begin{array}{l}\text { Inhabited 1930s-1940s; heavily } \\
\text { selectively logged 1986-1990; } \\
\text { Research station } 1986 \text { - } 2003 .\end{array}$ & $\begin{array}{l}\text { Minimal selective logging } \\
\text { 1988-1989. Satellite research } \\
\text { camp 1988 - 2005; ANGAP } \\
\text { satellite camp 2005 - present. }\end{array}$ & $\begin{array}{l}\text { Not logged; satellite } \\
\text { research camp } 1990 \\
\text { present }\end{array}$ \\
\hline
\end{tabular}

Sources of data: *Overdorff 1991, †White et al. 1995, ^Balko 1998, §Balko and Underwood 2005, †Arrigo-Nelson 2006. 


\section{CHAPTER II: A Description of Allobosca crassipes (Hippoboscidae: Diptera)}

\section{INTRODUCTION}

Flies of the family Hippoboscidae, commonly known as louse flies or keds, are parasites of a variety of birds and mammals. This family comprises 213 species, with 21 genera divided into 3 subfamilies (Dick 2006). These flies are patchily distributed, with locality records world-wide except from the polar regions (Maa 1969). Hippoboscids exhibit an unusual reproductive strategy with respect to most Diptera in that the females do not lay eggs. Instead, the females hold the larvae in their abdomen until they develop as pupae which are then deposited on the substrate, in proximity of or on the host (Bequaert 1953). Many louse flies are excellent fliers, one example being Ornithophila gestroi (Rondani) which has been observed pursuing falcons in flight, even in steep, high-speed dives (Balgooyen et al. 1999). Louse flies that parasitize mammals, such as the sheep ked Melophagus ovinus (Linnaeus), are flightless and exhibit vestigial wings so reduced that they are virtually wingless (Ferris \& Cole 1922). The hippoboscid that is the subject of this thesis, Allobosca crassipes (Speiser), exhibits an intermediate winged state in that it most probably emerges fully winged but loses the basal portion of its wings soon after acquiring a host (Theodor \& Oldroyd 1965).

The louse fly A. crassipes (Speiser) was first described and named by Paul Speiser in 1899. It is the monospecific representative of the genus Allobosca, subfamily Alloboscinae in the family Hippoboscidae, Order Diptera. It is an obligate parasite of lemurs in Madagascar and has traditionally been a little collected species (Maa 1969). 
The type description of A. crassipes was based on examination of three dried specimens obtained from "C. Hilger in Karlsruhe, Germany" (Speiser 1899:197). Speiser's (1899) original description of A. crassipes does not detail specific host or locality information from the specimens observed, mentioning only that the flies were collected from lemurs in Madagascar.

The type specimen is presumed destroyed during the bombings of Dresden in February of 1945 (Evenhuis, N. 2009. Personal communication). The initial description by Speiser and subsequent descriptions by Ferris \& Cole (1922) and Theodor \& Oldroyd (1965) were based from observations of nine total specimens which were in relatively poor condition. As no original material exists, the focus of this chapter is to re-describe and designate a neotype specimen for A. crassipes using specimens collected during San Jose State University expeditions in 2000-2001. The modern curation methods and the large sample size collected during the SJSU expeditions allows for consideration of intraspecific variation in A. crassipes as well.

\section{MATERIALS AND METHODS}

Live specimens of A. crassipes were collected from two species of lemurs, Varecia variegata variegata and Propithecus edwardsi in Talatekely (Tala), Vatoharanana (Vato) and Valohoaka (Valo), in Ranomafana National Park (RNP), Madagascar during field expeditions in 2000-2001. Lemur hosts $(n=29)$ were darted and tranquilized using Telazol ${ }$, with all collection methods in accordance with the protocol approved by San Jose State University's Institutional Animal Care and Use 
Committee (SJSU IACUC \#728). Fly specimens ( $\mathrm{n}=101)$ were collected within 30 minutes of capture of the hosts using forceps and a flea comb, and preserved in $80 \%$ ETOH. Precautions to prevent contamination by flies from different prosimian hosts included separation and isolation of lemurs prior and during ectoparasite collection. Cetylcide II was used to cleanse and disinfect all examination surfaces.

\section{Specimen Depository}

All specimens studied are deposited in the J. Gordon Edwards Museum of Entomology (JGEME) at San Jose State University, San Jose, California, 95192-0100, USA.

\section{RESULTS}

A total of 101 specimens were collected during the SJSU 2000-2001 expeditions to RNP (Table 2). All specimens, including the proposed neotype, are associated with lemurs that have been identified and tracked by lemur researchers since the 1980s (Pochron et al. 2004).

\section{Material Studied}

\section{Description}

The description of A. crassipes below is based on Speiser (1899), Ferris \& Cole (1922) and Theodor \& Oldroyd (1965), in addition to my own observations. Several anatomical features of A. crassipes are distinct. The head is rounded, well developed and easily viewed from the dorsal aspect (Figure 2). It is distinctly separated from the thorax and can move about freely. The eyes are well developed, and the antennae present a 
Table 2: List of Flies by Individual Lemur Host

\begin{tabular}{|c|c|c|c|c|c|c|c|}
\hline \multirow[b]{2}{*}{ Locality } & \multicolumn{4}{|c|}{ Lemurs } & \multicolumn{3}{|c|}{ Parasites } \\
\hline & \begin{tabular}{|c|} 
Host \\
Species
\end{tabular} & Host ID & $\begin{array}{l}\text { Host } \\
\text { Sex }\end{array}$ & $\begin{array}{c}\text { Host } \\
\text { Weight }\end{array}$ & $\begin{array}{c}\text { Fly Accession } \\
\text { Number }\end{array}$ & $\begin{array}{l}\text { Fly } \\
\text { Sex }\end{array}$ & $\begin{array}{c}\text { Number of } \\
\text { Flies on } \\
\text { Host }\end{array}$ \\
\hline$\overline{\text { Vato }}$ & VVV & Blue Green Grp II & male & $5.7 \mathrm{~kg}$ & $2000.14 \mathrm{AF}$ & $\bar{F}$ & 3 flies \\
\hline Vato & VVV & Blue Green Grp II & male & $5.7 \mathrm{~kg}$ & 2000.14BM & M & 3 flies \\
\hline Vato & VVV & Blue Green Grp II & male & $5.7 \mathrm{~kg}$ & 2000.14CM & M & 3 flies \\
\hline Vato & VVV & Red Gold Grp II & male & $3.4 \mathrm{~kg}$ & 2001.21AF & $\mathrm{F}$ & 3 flies \\
\hline Vato & VVV & Red Gold Grp II & male & $3.4 \mathrm{~kg}$ & 2001.21BF & $\mathrm{F}$ & 3 flies \\
\hline Vato & VVV & Red Gold Grp II & male & $3.4 \mathrm{~kg}$ & 2001.21CM & M & 3 flies \\
\hline Vato & VVV & Yellow Green & male & $3.3 \mathrm{~kg}$ & 2001.31AM & M & 2 flies \\
\hline Vato & VVV & Yellow Green & male & $3.3 \mathrm{~kg}$ & 2001.31BF & $\mathrm{F}$ & 2 flies \\
\hline Vato & VVV & Radio I & male & $3.0 \mathrm{~kg}$ & 2001.24AM & M & 2 flies \\
\hline Vato & VVV & Radio I & male & $3.0 \mathrm{~kg}$ & 2001.24BF & F & 2 flies \\
\hline Vato & VVV & Red Blue & female & $4.05 \mathrm{~kg}$ & 2001.27AF & $\mathrm{F}$ & 2 flies \\
\hline Vato & VVV & Red Blue & female & $4.05 \mathrm{~kg}$ & 2001.27BF & $\mathrm{F}$ & 2 flies \\
\hline Vato & VVV & Blue Green Grp I & male & $3.85 \mathrm{~kg}$ & 2001.28AF & $\mathrm{F}$ & 2 flies \\
\hline Vato & VVV & Blue Green Grp I & male & 3.85 kg & 2001.28BF & $\mathrm{F}$ & 2 flies \\
\hline Tala & PE & Turquoise & male & $4.8 \mathrm{~kg}$ & 2000.6AF & $\mathrm{F}$ & 1 fly \\
\hline Tala & $\mathrm{PE}$ & Blue Blue & female & $5.3 \mathrm{~kg}$ & 2000.4AF & $\mathrm{F}$ & 7 flies \\
\hline Tala & $\mathrm{PE}$ & Blue Blue & female & $5.3 \mathrm{~kg}$ & 2000.4BM & M & 7 flies \\
\hline Tala & $\mathrm{PE}$ & Blue Blue & female & $5.3 \mathrm{~kg}$ & $2000.4 \mathrm{CM}$ & M & 7 flies \\
\hline Tala & $\mathrm{PE}$ & Blue Blue & female & $5.3 \mathrm{~kg}$ & 2000.4DM & M & 7 flies \\
\hline Tala & $\mathrm{PE}$ & Blue Blue & female & $5.3 \mathrm{~kg}$ & 2000.4EM & M & 7 flies \\
\hline Tala & $\mathrm{PE}$ & Blue Blue & female & $5.3 \mathrm{~kg}$ & $2000.4 \mathrm{FM}$ & M & 7 flies \\
\hline Tala & $\mathrm{PE}$ & Blue Blue & female & $5.3 \mathrm{~kg}$ & $2000.4 \mathrm{GM}$ & M & 7 flies \\
\hline Tala & $\mathrm{PE}$ & Blue Green Grp IV & female & $5.2 \mathrm{~kg}$ & 2000.2AM & M & 2 flies \\
\hline Tala & $\mathrm{PE}$ & Blue Green Grp IV & female & $5.2 \mathrm{~kg}$ & 2000.2BM & M & 2 flies \\
\hline Tala & $\mathrm{PE}$ & Blue Purple & male & $5.3 \mathrm{~kg}$ & 2000.5AF & $\mathrm{F}$ & 6 flies \\
\hline Tala & $\mathrm{PE}$ & Blue Purple & male & $5.3 \mathrm{~kg}$ & 2000.5BF & $\mathrm{F}$ & 6 flies \\
\hline Tala & $\mathrm{PE}$ & Blue Purple & male & $5.3 \mathrm{~kg}$ & $2000.5 \mathrm{CF}$ & $\mathrm{F}$ & 6 flies \\
\hline Tala & $\mathrm{PE}$ & Blue Purple & male & $5.3 \mathrm{~kg}$ & 2000.5DF & $\mathrm{F}$ & 6 flies \\
\hline Tala & $\mathrm{PE}$ & Blue Purple & male & $5.3 \mathrm{~kg}$ & 2000.5EM & M & 6 flies \\
\hline Tala & $\mathrm{PE}$ & Blue Purple & male & $5.3 \mathrm{~kg}$ & 2000.5FM & M & 6 flies \\
\hline Tala & $\mathrm{PE}$ & no collar & female & $4.5 \mathrm{~kg}$ & 2000.9AF & $\mathrm{F}$ & 9 flies \\
\hline Tala & $\mathrm{PE}$ & no collar & female & $4.5 \mathrm{~kg}$ & 2000.9BF & $\mathrm{F}$ & 9 flies \\
\hline Tala & $\mathrm{PE}$ & no collar & female & $4.5 \mathrm{~kg}$ & $2000.9 \mathrm{CF}$ & $\mathrm{F}$ & 9 flies \\
\hline Tala & $\mathrm{PE}$ & no collar & female & $4.5 \mathrm{~kg}$ & 2000.9DF & $\mathrm{F}$ & 9 flies \\
\hline Tala & $\mathrm{PE}$ & no collar & female & $4.5 \mathrm{~kg}$ & 2000.9EF & $\mathrm{F}$ & 9 flies \\
\hline Tala & $\mathrm{PE}$ & no collar & female & $4.5 \mathrm{~kg}$ & 2000.9FM & M & 9 flies \\
\hline Tala & PE & no collar & female & $4.5 \mathrm{~kg}$ & 2000.9GM & M & 9 flies \\
\hline Tala & PE & no collar & female & $4.5 \mathrm{~kg}$ & $2000.9 \mathrm{HM}$ & M & 9 flies \\
\hline Tala & $\mathrm{PE}$ & no collar & female & $4.5 \mathrm{~kg}$ & 2000.9IM & M & 9 flies \\
\hline Tala & $\mathrm{PE}$ & Pink Grp IV & male & $5.4 \mathrm{~kg}$ & 2000.1AF & F & 4 flies \\
\hline Tala & $\mathrm{PE}$ & Pink Grp IV & male & $5.4 \mathrm{~kg}$ & $2000.1 \mathrm{BM}$ & M & 4 flies \\
\hline Tala & $\mathrm{PE}$ & Pink Grp IV & male & $5.4 \mathrm{~kg}$ & $2000.1 \mathrm{CM}$ & M & 4 flies \\
\hline Tala & PE & Pink Grp IV & male & $5.4 \mathrm{~kg}$ & 2000.1DM & M & 4 flies \\
\hline
\end{tabular}


Table 2: List of Flies by Individual Lemur Host (Continued)

\begin{tabular}{|c|c|c|c|c|c|c|c|}
\hline & \multicolumn{4}{|c|}{ Lemurs } & \multicolumn{3}{|c|}{ Parasites } \\
\hline Locality & \begin{tabular}{|c|} 
Host \\
Species
\end{tabular} & Host ID & $\begin{array}{l}\text { Host } \\
\text { Sex }\end{array}$ & $\begin{array}{c}\text { Host } \\
\text { Weight }\end{array}$ & $\begin{array}{c}\text { Fly Accession } \\
\text { Number }\end{array}$ & $\begin{array}{l}\text { Fly } \\
\text { Sex }\end{array}$ & $\begin{array}{c}\text { Number of } \\
\text { Flies on } \\
\text { Host }\end{array}$ \\
\hline Tala & $\overline{\mathrm{PE}}$ & Purple Grp IV & male & $4.9 \mathrm{~kg}$ & 2000.3AM & $\mathrm{M}$ & 2 flies \\
\hline Tala & $\mathrm{PE}$ & Purple Grp IV & male & $4.9 \mathrm{~kg}$ & 2000.3BM & M & 2 flies \\
\hline Tala & $\mathrm{PE}$ & Blue Grp IV & male & $5.3 \mathrm{~kg}$ & 2001.17AM & M & 1 fly \\
\hline Tala & PE & Green Orange Grp & I male & $5.9 \mathrm{~kg}$ & 2001.16AM & M & 4 flies \\
\hline Tala & $\mathrm{PE}$ & Green Orange Grp & I male & $5.9 \mathrm{~kg}$ & 2001.16BF & F & 4 flies \\
\hline Tala & PE & Green Orange Grp & I male & $5.9 \mathrm{~kg}$ & 2001.16CF (type) & $\mathrm{F}$ & 4 flies \\
\hline Tala & $\mathrm{PE}$ & Green Orange Grp & I male & $5.9 \mathrm{~kg}$ & 2001.16DF & $\mathrm{F}$ & 4 flies \\
\hline Tala & PE & no collar Grp I & male & $2.9 \mathrm{~kg}$ & 2001.15AF & $\mathrm{F}$ & 2 flies \\
\hline Tala & PE & no collar Grp I & male & $2.9 \mathrm{~kg}$ & 2001.15BF & F & 2 flies \\
\hline Vato & $\mathrm{PE}$ & Red Grp I & male & $5.3 \mathrm{~kg}$ & 2000.10AM & M & $1 \mathrm{fly}$ \\
\hline Vato & $\mathrm{PE}$ & Green Gold Grp III & female & $5.5 \mathrm{~kg}$ & 2000.11AM & M & 2 flies \\
\hline Vato & $\mathrm{PE}$ & Green Gold Grp III & female & $5.5 \mathrm{~kg}$ & 2000.11BF & $\mathrm{F}$ & 2 flies \\
\hline Vato & PE & Purple Gold Grp II & male & $5.4 \mathrm{~kg}$ & 2000.12AM & M & 2 flies \\
\hline Vato & $\mathrm{PE}$ & Purple Gold Grp II & male & $5.4 \mathrm{~kg}$ & 2000.12BM & M & 2 flies \\
\hline Vato & $\mathrm{PE}$ & Yellow Blue Grp II & I female & $5.6 \mathrm{~kg}$ & 2000.13AF & F & 7 flies \\
\hline Vato & $\mathrm{PE}$ & Yellow Blue Grp II & I female & $5.6 \mathrm{~kg}$ & 2000.13BF & $\mathrm{F}$ & 7 flies \\
\hline Vato & $\mathrm{PE}$ & Yellow Blue Grp II & I female & $5.6 \mathrm{~kg}$ & 2000.13CF & $\mathrm{F}$ & 7 flies \\
\hline Vato & $\mathrm{PE}$ & Yellow Blue Grp II & f female & $5.6 \mathrm{~kg}$ & $2000.13 \mathrm{DF}$ & $\mathrm{F}$ & 7 flies \\
\hline Vato & $\mathrm{PE}$ & Yellow Blue Grp II & I female & $5.6 \mathrm{~kg}$ & $2000.13 \mathrm{EF}$ & $\mathrm{F}$ & 7 flies \\
\hline Vato & PE & Yellow Blue Grp Il & I female & $5.6 \mathrm{~kg}$ & 2000.13FM & M & 7 flies \\
\hline Vato & PE & Yellow Blue Grp Il & I female & $5.6 \mathrm{~kg}$ & 2000.13GM & M & 7 flies \\
\hline Vato & PE & Brown Blue Grp II & male & $5.8 \mathrm{~kg}$ & 2001.23AF & $\mathrm{F}$ & 4 flies \\
\hline Vato & $\mathrm{PE}$ & Brown Blue Grp II & male & $5.8 \mathrm{~kg}$ & 2001.23BF & F & 4 flies \\
\hline Vato & $\mathrm{PE}$ & Brown Blue Grp II & male & $5.8 \mathrm{~kg}$ & 2001.23CM & M & 4 flies \\
\hline Vato & $\mathrm{PE}$ & Brown Blue Grp II & male & $5.8 \mathrm{~kg}$ & 2001.23DM & M & 4 flies \\
\hline Vato & $\mathrm{PE}$ & Green Gold III & male & $5.0 \mathrm{~kg}$ & 2001.22AF & $\mathrm{F}$ & 4 flies \\
\hline Vato & $\mathrm{PE}$ & Green Gold III & male & $5.0 \mathrm{~kg}$ & 2001.22BM & M & 4 flies \\
\hline Vato & PE & Green Gold III & male & $5.0 \mathrm{~kg}$ & 2001.22CM & M & 4 flies \\
\hline Vato & $\mathrm{PE}$ & Green Gold III & male & $5.0 \mathrm{~kg}$ & 2001.22DM & M & 4 flies \\
\hline Vato & $\mathrm{PE}$ & Green silver I & male & $3.5 \mathrm{~kg}$ & 2001.26AM & M & 6 flies \\
\hline Vato & $\mathrm{PE}$ & Green silver I & male & $3.5 \mathrm{~kg}$ & 2001.26BM & M & 6 flies \\
\hline Vato & $\mathrm{PE}$ & Green silver I & male & $3.5 \mathrm{~kg}$ & 2001.26CF & $\mathrm{F}$ & 6 flies \\
\hline Vato & $\mathrm{PE}$ & Green silver I & male & $3.5 \mathrm{~kg}$ & 2001.26DF & $\mathrm{F}$ & 6 flies \\
\hline Vato & $\mathrm{PE}$ & Green silver I & male & $3.5 \mathrm{~kg}$ & 2001.26EF & $\mathrm{F}$ & 6 flies \\
\hline Vato & $\mathrm{PE}$ & Green silver I & male & $3.5 \mathrm{~kg}$ & 2001.26FF & $\mathrm{F}$ & 6 flies \\
\hline Vato & $\mathrm{PE}$ & Green silver red II & male & $3.05 \mathrm{~kg}$ & 2001.20AM & M & 1 fly \\
\hline Vato & $\mathrm{PE}$ & Pink silver II & male & $4.60 \mathrm{~kg}$ & 2001.19AM & M & 6 flies \\
\hline Vato & $\mathrm{PE}$ & Pink silver II & male & $4.60 \mathrm{~kg}$ & 2001.19BM & M & 6 flies \\
\hline Vato & PE & Pink silver II & male & $4.60 \mathrm{~kg}$ & 2001.19CM & M & 6 flies \\
\hline Vato & $\mathrm{PE}$ & Pink silver II & male & $4.60 \mathrm{~kg}$ & 2001.19DM & M & 6 flies \\
\hline Vato & $\mathrm{PE}$ & Pink silver II & male & $4.60 \mathrm{~kg}$ & 2001.19EM & M & 6 flies \\
\hline Vato & PE & Pink silver II & male & $4.60 \mathrm{~kg}$ & 2001.19FF & $\mathrm{F}$ & 6 flies \\
\hline
\end{tabular}


Table 2: List of Flies by Individual Lemur Host (Continued)

\begin{tabular}{|c|c|c|c|c|c|c|c|}
\hline & \multicolumn{4}{|c|}{ Lemurs } & \multicolumn{3}{|c|}{ Parasites } \\
\hline Locality & \begin{tabular}{|c|} 
Host \\
Species
\end{tabular} & Host ID & $\begin{array}{l}\text { Host } \\
\text { Sex }\end{array}$ & $\begin{array}{c}\text { Host } \\
\text { Weight }\end{array}$ & $\begin{array}{c}\text { Fly Accession } \\
\text { Number }\end{array}$ & $\begin{array}{l}\text { Fly } \\
\text { Sex }\end{array}$ & $\begin{array}{c}\text { Number of } \\
\text { Flies on } \\
\text { Host }\end{array}$ \\
\hline Vato & $\mathrm{PE}$ & Purple blue I & male & $5.4 \mathrm{~kg}$ & 2001.29AF & $\mathrm{F}$ & 4 flies \\
\hline Vato & $\mathrm{PE}$ & Purple blue I & male & $5.4 \mathrm{~kg}$ & 2001.29BF & $\mathrm{F}$ & 4 flies \\
\hline Vato & $\mathrm{PE}$ & Purple blue I & male & $5.4 \mathrm{~kg}$ & 2001.29CF & F & 4 flies \\
\hline Vato & $\mathrm{PE}$ & Purple blue I & male & $5.4 \mathrm{~kg}$ & 2001.29DF & $\mathrm{F}$ & 4 flies \\
\hline Vato & $\mathrm{PE}$ & Radio I & male & $5.35 \mathrm{~kg}$ & 2001.25AF & F & 9 flies \\
\hline Vato & $\mathrm{PE}$ & Radio I & male & $5.35 \mathrm{~kg}$ & 2001.25BF & F & 9 flies \\
\hline Vato & $\mathrm{PE}$ & Radio I & male & $5.35 \mathrm{~kg}$ & 2001.25CF & $\mathrm{F}$ & 9 flies \\
\hline Vato & PE & Radio I & male & $5.35 \mathrm{~kg}$ & 2001.25DM & M & 9 flies \\
\hline Vato & PE & Radio I & male & $5.35 \mathrm{~kg}$ & 2001.25EM & M & 9 flies \\
\hline Vato & $\mathrm{PE}$ & Radio I & male & $5.35 \mathrm{~kg}$ & 2001.25FM & M & 9 flies \\
\hline Vato & $\mathrm{PE}$ & Radio I & male & $5.35 \mathrm{~kg}$ & 2001.25GM & M & 9 flies \\
\hline Vato & PE & Radio I & male & $5.35 \mathrm{~kg}$ & 2001.25HM & M & 9 flies \\
\hline Vato & PE & Radio I & male & $5.35 \mathrm{~kg}$ & 2001.25IM & M & 9 flies \\
\hline Vato & $\mathrm{PE}$ & Radio II & male & $5.90 \mathrm{~kg}$ & 2001.18AM & M & $1 \mathrm{fly}$ \\
\hline Valo & PE & Pink blue IV & female & $6.25 \mathrm{~kg}$ & 2001.30AM & M & 2 flies \\
\hline Valo & PE & Pink blue IV & female & $6.25 \mathrm{~kg}$ & 2001.30BM & M & 2 flies \\
\hline
\end{tabular}




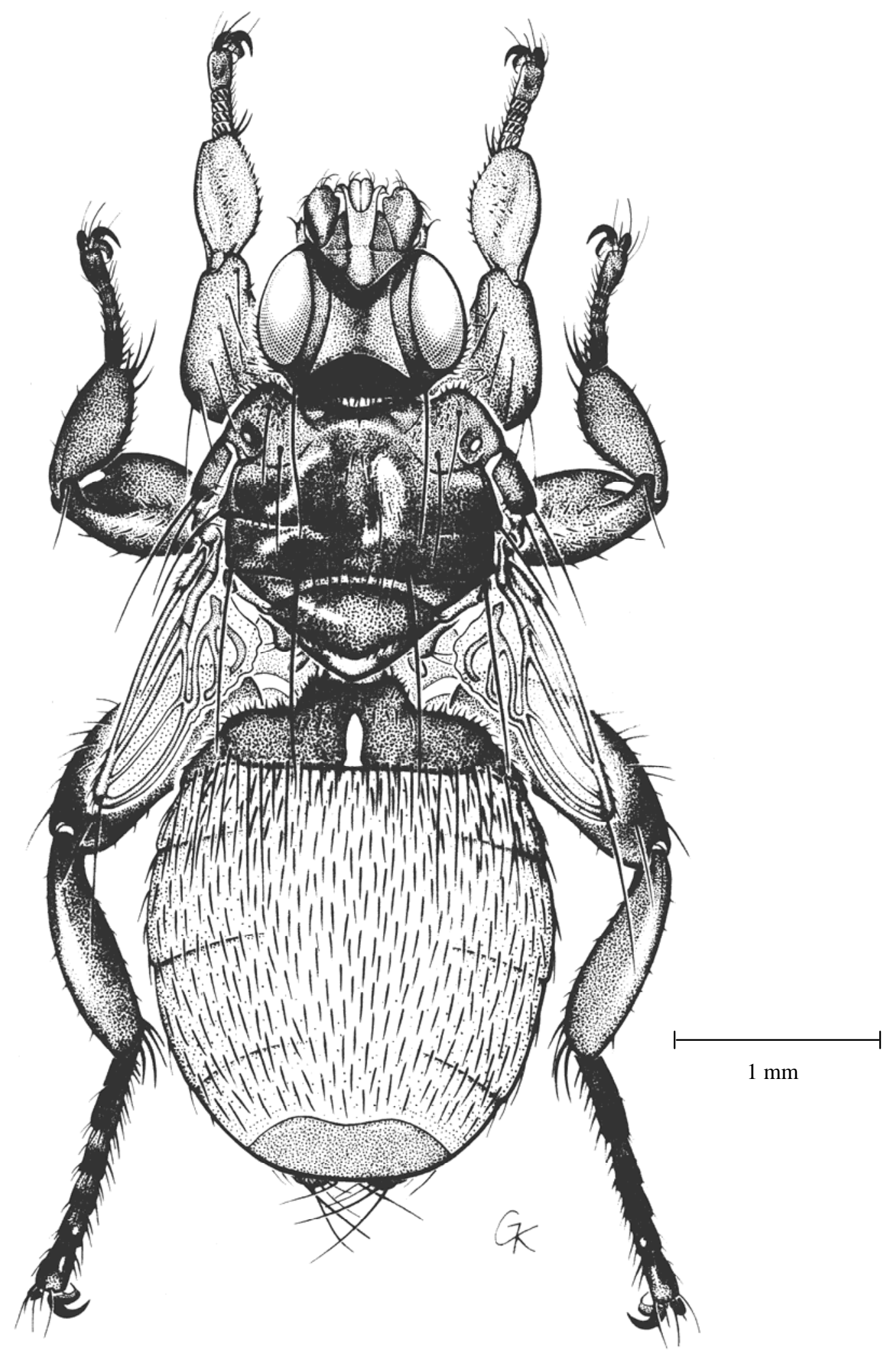

Figure 2. Allobosca crassipes, male, dorsal view. (Illustration by Ginny Kirsch.) 
dorsal process and a lobed, leaf-shaped arista. The fore femora are strongly swollen basally and the front tarsi differ from the mid and hind legs in exhibiting short, nearly spatulate setae on the first four segments. The wings, when present, usually have seven longitudinal veins, all heavily scleritized, with three cross veins in line with each other. The veins making up the discal and anal cells may or may not be entire or complete, depending on the extent of the shedding of the basal portion of the wing. The discal and anal cells generally are triangular in shape and nearly equal in size (Figures 3A \& 3B). There are no other distinctive features of the thoracic region useful for differentiating $A$. crassipes from any other related species.

Included in Theodor \& Oldroyd's (1965) description of A. crassipes, was an illustration of a wing with a substantial portion of the basal membrane still present. This is illustrated in Figure 3A. Observations of the specimens collected during the SJSU 2000-2001 expeditions did not reveal such a complete remnant of this membrane. A typical wing of the flies collected from RNP, with the basal portion more completely shed, is shown in Figure 3B for comparison.

\section{Sexing Adult Flies}

The external structure and morphology of the adult male and female are essentially the same. The only difference of note is some degree of sexual dimorphism; the male is generally smaller in size and the abdomen is clearly rounded (Figure 4A). The morphology of the female abdomen presents a distinct bilobed terminex as shown in Figure 4B. The specimen proposed as the neotype is an adult female, designated here as 


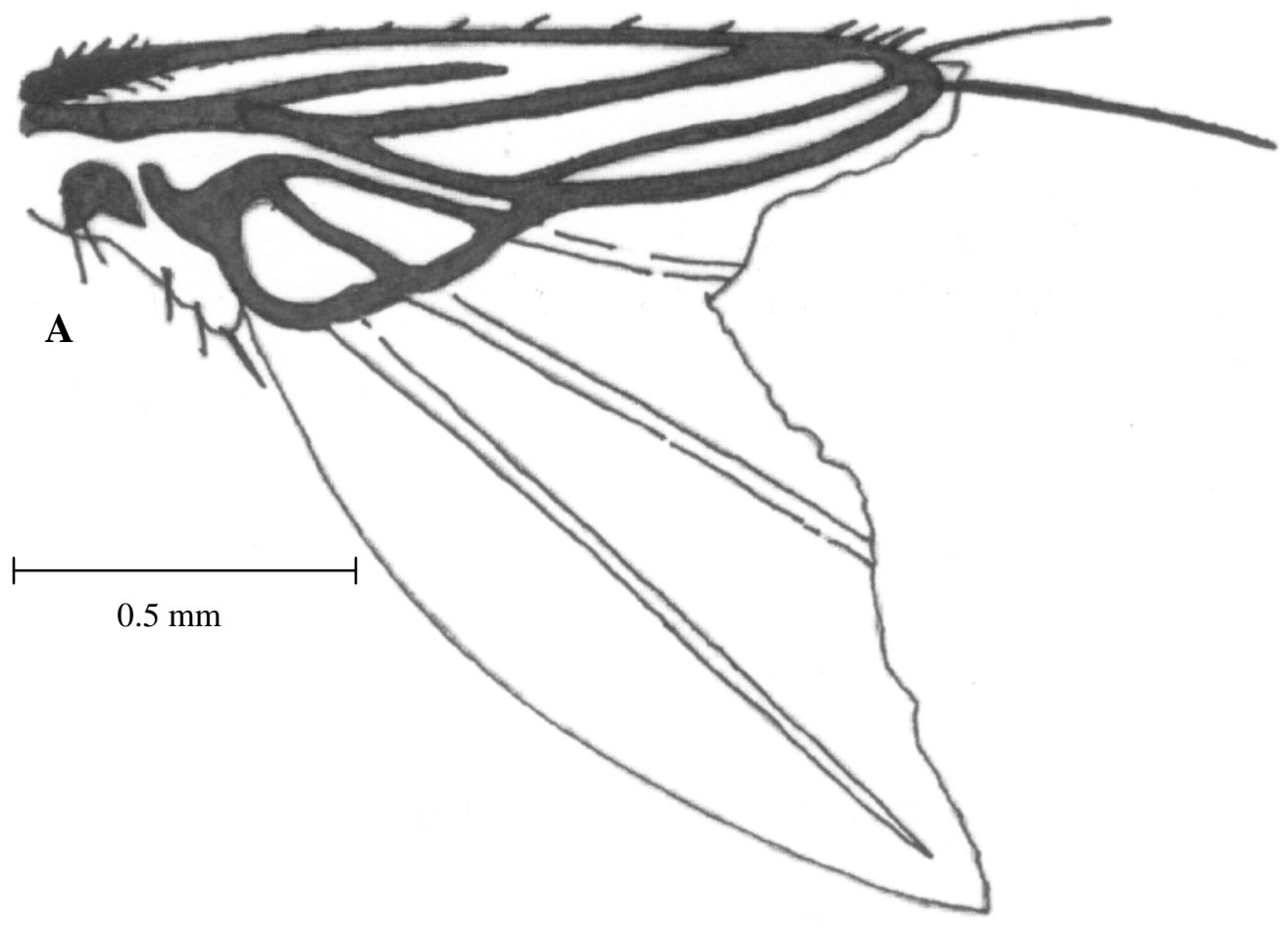

B

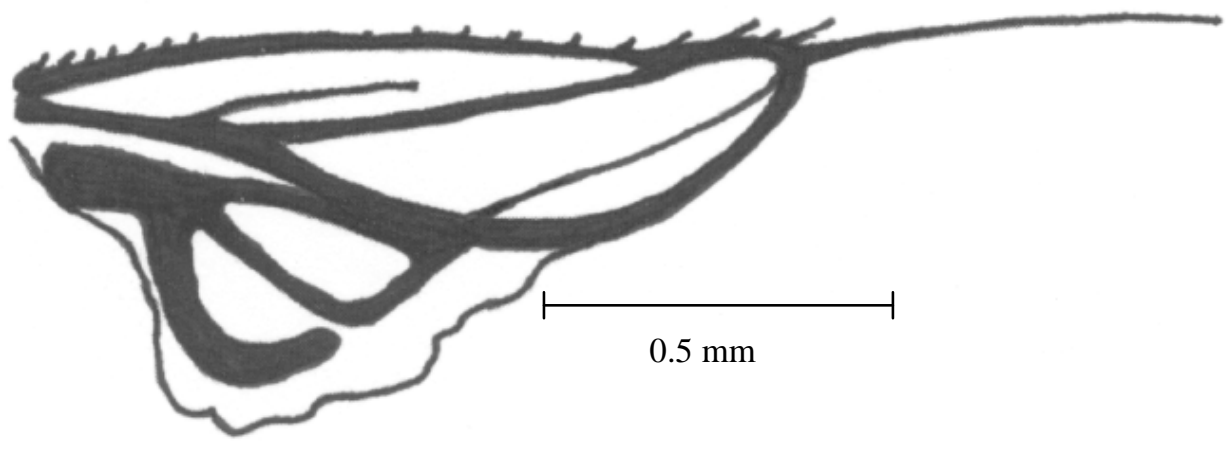

Figure 3. A. crassipes wing; (A) with basal portion partially intact (after Theodor \& Oldroyd 1965); (B) with basal portion of the wing shed (Specimen 2000.9BF). 

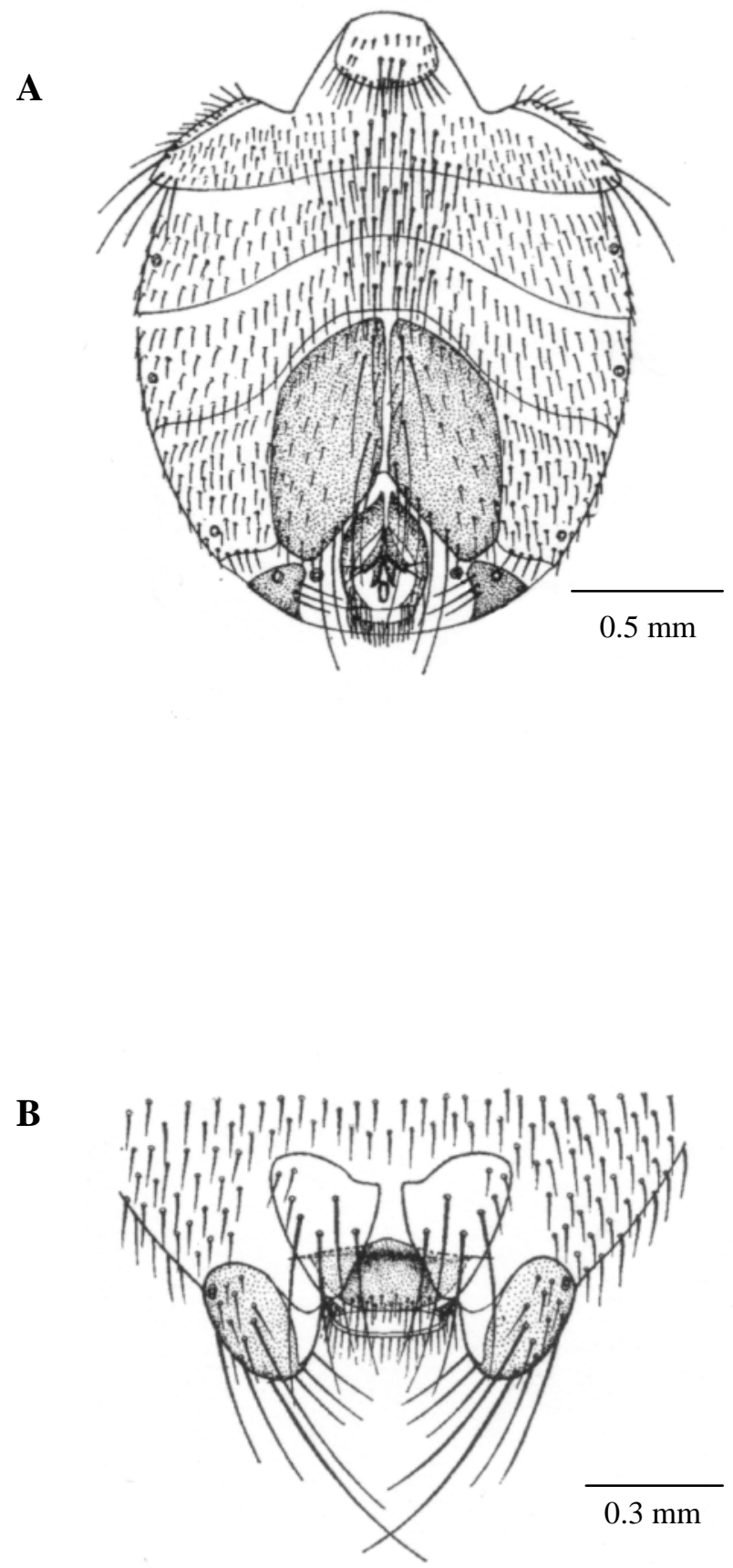

Figure 4. (A) A. crassipes, abdomen, male, ventral; (B) A. crassipes, posterior part of abdomen, female, ventral. From Theodor \& Oldroyd (1965:95-96). 
2001.16CF; collected on 7 November 2001 from P. edwardsi (individual "Green Orange" group I, Talatekely Trail System), Ranomafana National Park, Madagascar.

\section{DISCUSSION}

The description presented above uses the key morphological features discussed by Speiser (1899), Ferris \& Cole (1922), Bequaert (1952), and Theodor \& Oldroyd (1965). There are some differences, however, with respect to some of the morphological features used by previous researchers and the observation of these features from specimens collected during SJSU expeditions in 2000-2001. For example, Ferris \& Cole (1922) incorrectly report that both sexes of A. crassipes possess a single, large scleritized abdominal plate. This is inaccurate as only the female presents a single large scleritzed abdominal plate. The male exhibits two distinct, oval abdominal plates of equal size. This difference, first noted by Theodor \& Oldroyd (1965), is consistent with observations of the specimens collected during the SJSU expeditions.

An abdominal feature not previously used in describing the structural differences between male and female flies is the relative density of setae found on the $5^{\text {th }}$ abdominal sternite and the thickness of the individual setae in this region. Observations of the setae found on the $5^{\text {th }}$ abdominal sternite of males is generally denser and individual setal thickness is greater than that observed on the same region in females. Further, some noticeable variation in the density and thickness of these abdominal setae was observed in male specimens. For example, specimens 2000.1BM, 2001.19EM, and 2001.25IM exhibit considerably more densely distributed and thicker setae than specimen 2000.4EM. 
All other male specimens examined presented a setal density and thickness somewhere in between these extremes. Comparable variation of setal density and thickness was not observed in the female specimens collected.

Previous descriptions of the wings of A. crassipes indicate shedding of the basal portion, leaving a stump with radial veins $R_{1}$ to $R_{4+5}$, the basal sections of medial veins $\mathrm{M}_{1+2}$ and $\mathrm{M}_{3+4}$ and the anal cell heavily sclerotized (Theodor \& Oldroyd 1965).

Although this description is consistent with the observations made of the specimens collected from RNP in 2000-2001, the anal cell was not observed to be entirely closed as had been previously reported (Theodor \& Oldroyd 1965). Figure 3 illustrates this observed difference.

The differences in the observations of Speiser (1899), Ferris \& Cole (1922) and Theodor \& Oldroyd (1965) may be attributed to the limited sample size and the less than ideal condition of the specimens examined. Speiser (1899) reports that the specimens he received were "dried", and Ferris \& Cole (1922) based their descriptions on specimens that were obtained from a single lemur skin housed in the National Museum (Smithsonian Institution) some 60 years after the lemur specimen was collected. In both cases, specific records of host and locality are incomplete. Prior to the SJSU study, the number and condition of the examined specimens were few in number (three by Speiser and six by Ferris \& Cole) and were degraded in comparison with the specimens from SJSU reported here. The modern collection and curation of the 101 specimens collected during SJSU field work in 2000-2001 provide the most comprehensive data set thus 
established for detailing key morphometric characters of A. crassipes, with sufficient sample size for further investigation and discussion of intraspecific variation. 


\section{CHAPTER III: Association of Allobosca crassipes (Diptera: Hippoboscidae) with the Black and White Ruffed Lemur (Varecia variegata variegata) and Milne- Edwards' Sifaka (Propithecus edwardsi) in Southeastern Madagascar*}

\section{INTRODUCTION}

Field research on the relationship between lemurs and their ectoparasites in Ranomafana National Park (RNP, $21^{\circ} 16^{\prime} \mathrm{S}, 47^{\circ} 20^{\prime}$ E, elevation 900-1200m) in southeastern Madagascar has resulted in a new association of the hippoboscid Allobosca crassipes (Speiser, 1899) with the black and white ruffed lemur (Varecia variegata variegata (Kerr, 1792)) and a confirmed association with Milne-Edwards' sifaka (Propithecus edwardsi (Grandidier, 1871)). Previously, Maa (1969) noted host records for the lemurids Eulemur macaco (Linnaeus, 1766) and E. rubriventer (I. Geoffroy, 1850), the lepilemurid Lepilemur mustelinus (I. Geoffroy, 1851), and the indriids Avahi laniger (Gmelin, 1788) and Propithecus diadema (Bennett, 1832). Ferris \& Cole (1922) collected six specimens of A. crassipes from a skin of Propithecus edwardsi housed in the collections of the United States National Museum, but did not provide specific locality or specimen information of the host, and the repository of the six flies is unstated and currently unknown.

\section{MATERIALS AND METHODS}

A. crassipes, an obligate ectoparasite restricted to Madagascar, historically has been a little collected species. During San Jose State University expeditions in 20002001, individuals of two lemur species ( $V$. variegata variegata and P. edwardsi) were

\footnotetext{
* This chapter appeared in Pan-Pacific Entomologist 85:162-166.
} 
tranquilized as part of a larger study investigating the biogeochemical effects of anthropogenic disturbance in lemur populations within RNP (McGee \& Vaughn 2007, McGee et al. 2008). Collection methods are in accordance with the protocol approved by San Jose State University's Institutional Animal Care and Use Committee (SJSU IACUC \#728). A total of 101 specimens of $A$. crassipes were obtained from $V$. variegata variegata and P. edwardsi. Ectoparasites were collected within 30 minutes of capture using forceps and a flea comb. Specimens were preserved in $80 \% \mathrm{ETOH}$. Precautions to prevent host contamination by ectoparasites from different individuals included separation and isolation of lemurs prior, during and after ectoparasite collection. Cetylcide II was used to cleanse and disinfect all examination surfaces.

\section{RESULTS}

On 7 December 2000, three hippoboscids were collected from an immature male of $V$. variegata variegata (identified by University of Texas banding collar as Blue Green Group II, a male weighing $5.7 \mathrm{~kg}$ ) from the Vatoharanana trail system in RNP. The following year, 11 additional hippoboscids were collected between 13 November and 17 November 2001 from five individuals of V. variegata variegata: Red Gold II, male, 3.4 kg (3 flies); Yellow Green, male, 3.3 kg (2 flies); Radio I, male, 3.0 kg (2 flies); Red Blue, female, $4.05 \mathrm{~kg}$ (2 flies); and Blue Green I, male, $3.85 \mathrm{~kg}$ (2 flies) also from the Vatoharanana trail system. A total of 5 male and 9 female flies were collected from $V$. variegata variegata.

The 2000-2001 expeditions also yielded 87 specimens of $A$. crassipes from 
another species of lemur, P. edwardsi, in RNP. These confirm a host record that previously was noted only from a single museum specimen of $P$. edwardsi by Ferris \& Cole (1922).

Since Speiser's (1899) initial identification of an association of A. crassipes with the diademed sifaka, P. diadema, and Ferris \& Cole's (1922) subsequent identification of A. crassipes with P. edwardsi, the taxonomy of Propithecus has undergone significant reinterpretation and rearrangement, which may lead to some confusion about host records. P. diadema and P. edwardsi were initially described as separate species in the 1800 s by Bennett (1832) and Grandidier (1871), respectively. Petter et al. (1977) and Tattersall (1982), however, considered the diademed sifaka and Milne-Edwards' sifaka as polytypics of a single species, $P$. diadema. Following Tattersall (1986), four subspecies of $P$. diadema were recognized ( $P$. diadema diadema, $P$. diadema edwardsi, $P$. diadema candidus (Grandidier, 1871), and P. diadema perrieri (Lavauden, 1931)), each distinct phenotypes with nonoverlapping ranges in eastern and northern Madagascar. The use of subspecific designations, however, predates Petter al al. (1977) and Tattersall (1982, 1986); one particularly noteworthy example comes from Bequaert (1952) who refers to an association of $A$. crassipes with P. diadema edwardsi. The subspecies designations of Propithecus remained in use until Mayor et al. (2004) gave all subspecies specific status on the basis of morphometric and genetic data. Groves and Helgen (2007) supported this classification with additional morphometric data. With P. diadema and P. edwardsi once again considered distinct species, the associations of Speiser (1899) and Ferris \& Cole (1922) are consistent with current taxonomic usage. 
Between 19 November and 29 November 2000, flies were collected from seven individuals of P. edwardsi from the Talatakely trail system: Turquoise, male, $4.8 \mathrm{~kg}$ (1 fly); Blue Blue, female, $5.3 \mathrm{~kg}$ (7 flies); Blue Green IV, female, $5.2 \mathrm{~kg}$ (2 flies); Blue Purple, male, 5.3 kg (6 flies); "No collar”, female, $4.5 \mathrm{~kg}$ (9 flies); Pink IV, male, $5.4 \mathrm{~kg}$ (4 flies); and Purple IV, male, $4.9 \mathrm{~kg}$ (2 flies). The following year on 11 November 2001, flies were collected from three individuals of P. edwardsi from the Talatakely trail system: Blue IV, male, $5.3 \mathrm{~kg}$ (1 fly); Green Orange I, male, $5.9 \mathrm{~kg}$ (4 flies); and "No collar I", male, $2.9 \mathrm{~kg}$ ( 2 flies). A total of 21 male and 17 female flies were collected from P. edwardsi at Talatakely.

A. crassipes was also collected from P. edwardsi along the Vatoharanana and Valohoaka trail systems in RNP. Between 27 November and 5 December 2000, 12 flies were collected from four individuals of $P$. edwardsi from the Vatoharanana trail system: Red I, male, 5.3 kg (1 fly); Green Gold III, female, 5.5 kg (2 flies); Purple Gold III, male, $5.4 \mathrm{~kg}$ (2 flies); and Yellow Blue III, female, $5.6 \mathrm{~kg}$ (7 flies). The following year, between 12 November and 16 November 2001, 35 flies were collected from eight individuals of P. edwardsi along the Vatoharanana trail system: Brown Blue III, male, 5.8 kg (4 flies); Green Gold III, male, $5.0 \mathrm{~kg}$ (4 flies); Green Silver I, male, $3.5 \mathrm{~kg}$ (6 flies); Green Silver Red II, male, 3.05 kg (1 fly); Pink Silver II, male, $4.60 \mathrm{~kg}$ (6 flies); Purple Blue I, male, $5.4 \mathrm{~kg}$ (4 flies); Radio I, male, $5.35 \mathrm{~kg}$ (9 flies); Radio II, male, 5.90 (1 fly). A total of 26 male and 21 female flies were collected from P. edwardsi at Vatoharanana. Lastly, on 8 November 2001, two specimens of A. crassipes were collected from an individual of P. edwardsi along the Valohoaka trail system: Pink Blue 
IV, female, $6.25 \mathrm{~kg}$ ( 2 flies). A total of two male flies (no females) were collected from P. edwardsi at Valohoaka.

\section{DISCUSSION}

The type specimens of A. crassipes referred to by Speiser (1899) are believed to have been destroyed during the World War II bombings of Dresden (N.L. Evenhuis, personal communication 2009). The 14 specimens taken from $V$. variegata variegata from Vatoharanana represent a new host record in Madagascar. The 87 specimens collected from P. edwardsi confirm an association previously identified and add to the record detailed information on localities and host animals. Collected specimens are housed at the J. Gordon Edwards Museum of Entomology at San Jose State University, San Jose, CA 95192-0100.

Maa (1963) commented that host specificity in hippoboscids is moderately high, noting in particular that louse flies are found on unrelated species in similar habitats. However, our documentation of the presence of A. crassipes on several species of related lemurs that vary in diet and habitat suggests a different type of host specificity of this parasite. The plasticity of the louse fly is remarkable given the differences between ruffed lemurs and sifakas. Ruffed lemurs and sifakas occur sympatrically in eastern Madagascar but have different niches. Ruffed lemurs are obligate frugivores and are patchily distributed in low densities in mid-latitude and lowland rainforests (Garbutt 1999). They are behaviorally and demographically highly sensitive to the availability of fruit, particularly in the dry season (Balko \& Underwood 2005). Sifakas, in contrast, rely 
less heavily on fruit, switching to leaves during the dry season (Balko \& Underwood 2005). Whereas elsewhere ruffed lemurs and sifakas inhabit primary and secondary forest, in Ranomafana National Park, the black and white ruffed lemur inhabits only primary forest. Milne-Edwards' sifaka is more broadly distributed, inhabiting both primary and disturbed forests. On the other hand, these species have comparable home ranges (i.e., distance traveled for food and cover), and at least half of the fruit species consumed by Milne-Edwards' sifaka are also consumed by species of Varecia (Dew \& Wright 1998, Arrigo-Nelson, 2006). Both V. variegata variagata and P. edwardsi have been observed to be geophagous (i.e., deliberately ingesting soil). Otherwise habitually arboreal, these lemurs descend to the ground on a regular basis to consume dirt (White 1989, Arrigo-Nelson et al. in press). A. crassipes most likely emerges from the pupa with fully developed wings, with the basal membrane lost after it locates its host (Theodor \& Oldroyd 1965). All flies collected exhibited significant wing reduction (i.e., are flightless), and therefore the geophagous behavior of $V$. variegata variegata and $P$. edwardsi affords the flies increased opportunities for contact and feeding on these hosts. Finally, differences due to habitat disturbance are likewise not likely to differentiate the distribution or abundance of A. crassipes. Wright et al. (2009) found that there was no significant difference in infection intensity of hippoboscids on P. edwardsi inhabiting disturbed and undisturbed forests in Ranomafana National Park: on average, 1.25 to 2.48 flies were found on P. edwardsi in Ranomafana. Of the two lemur species, V. variegata variagata has the most narrow or specialized dietary and habitat requirements. Future study might then focus on characteristics that are restrictive in the life history of the host 
species, i.e., in the case of $V$. variegata variagata, the distribution and phenology of fruiting tropical trees that may have played a role in the ecology and evolution of this host-parasite interaction. 


\section{CHAPTER IV: Effect of Habitat Disturbance on Stable Isotope Composition of Allobosca crassipes (Diptera: Hippoboscidae) from Southeastern Madagascar}

\section{INTRODUCTION}

A biodiversity hotspot is defined as a biogeographic region with a significant reservoir of biodiversity that is under threat from humans (Myers et al. 2000). Anthropogenic habitat disturbance in Madagascar is well documented, with over $90 \%$ of the native vegetation lost, mostly due to the practice of slash-and-burn agriculture (Wright 1992). This practice, as well as commercial logging, has occurred in areas that are within and around the boundaries of Ranomafana National Park (RNP), as recently as the late $1980 \mathrm{~s}$. Within RNP, about $50 \%$ of the vegetation has been altered by selective logging and other anthropogenic activities (Wright 1997). International teams have been studying the effects of anthropogenic disturbance on biodiversity in RNP for three decades (see Wright 1997). For example, since 2000, Dr. Elizabeth McGee (San Jose State University) has been looking at the effects of anthropogenic disturbance on the biogeochemistry of two lemurs, Proprithecus edwardsi and Varecia variegata variegata. As part of her analysis, she has used stable isotope analysis to develop a profile detailing carbon and nitrogen composition across habitats and through time. I suggest that anthropogenic disturbance will track biogeochemically between primary consumers (lemurs) and secondary consumers (louse flies). To quantify these effects, I employed the same analysis of stable carbon and nitrogen as was done by McGee \& Wright (2001), McGee \& Vaughn (2003, 2006, 2007), McGee et al. (2007, 2008). 


\section{STABLE ISOTOPE ANALYSIS}

Stable isotope analysis covers a broad spectrum of applications that take advantage of the phenomenon of fractionation between isotopes of the same element due to differences in mass. The use of stable isotopes was initially used by geophysicists (Hobson \& Wassenaar 1999) to compare differential changes in the ratios of isotopes to established standards to examine physical phenomena such as climate change, etc. (Burk \& Stuiver 1981; Yapp \& Epstein 1982). The earliest applications in ecology were from DeNiro \& Epstein (1978) and DeNiro \& Epstein (1981) who examined the influence of diet on the distribution of carbon isotopes and nitrogen isotopes in animals, respectively. This technique has also been used by researchers in archeology to reconstruct the diets of historic and prehistoric human populations (Ambrose \& DeNiro 1986) and in paleontology to identify the diets of extinct animals (Koch et al. 1994).

Regarding its ecological application, isotope analysis provides information about energy transfer in an ecosystem, i.e., the isotopic composition of organisms, yields important data that indicate how energy flows through the biosphere. Recent research suggests that the carbon and nitrogen isotope composition of primate hair is an indicator of habitat use and diet, respectively (Schoeninger et al. 1997, 1998). More recently, studies indicate that stable isotope analysis may be useful as a tool to track disturbance in rainforest ecosystems in that changes in isotope composition will track environmental changes (natural and anthropogenic) to the extent that habitat and diet are affected (McGee \& Wright 2001; McGee \& Vaughn 2003, 2006, 2007; McGee et al. 2007, 2008). 
Several elements occur as stable isotopes (Table 2). For the purpose of this study, the stable isotopes of carbon $\left({ }^{12} \mathrm{C}\right.$ and $\left.{ }^{13} \mathrm{C}\right)$ and nitrogen $\left({ }^{14} \mathrm{~N}\right.$ and $\left.{ }^{15} \mathrm{~N}\right)$ were used. Average terrestrial abundance (\%) for these elements is shown below in Table 3.

Table 3: Average Terrestrial Abundance for Carbon and Nitrogen

\begin{tabular}{ccc}
\hline Element & Isotope & $\begin{array}{c}\text { Average Terrestrial } \\
\text { Abundance (\%) }\end{array}$ \\
\hline \multirow{2}{*}{ Carbon } & ${ }^{12} \mathrm{C}$ & 98.980 \\
& ${ }^{13} \mathrm{C}$ & 1.110 \\
Nitrogen & ${ }^{14} \mathrm{~N}$ & 99.630 \\
& ${ }^{15} \mathrm{~N}$ & 0.370 \\
\hline
\end{tabular}

\section{Stable Nitrogen Isotopes}

Within animals stable nitrogen is an indicator of diet; specifically the relative amount of protein in an animal's diet. It has been observed that each increase in trophic level in the natural food chain results in a $3 \%$ enrichment in ${ }^{15} \mathrm{~N}$ (DeNiro \& Epstein 1981; Minagowa \& Wada 1984; Macko \& Engel 1991).

Isotopic composition is expressed as a ratio using the $\delta$ notation in parts per thousand (\%o). Nitrogen isotope composition is:

$$
\delta^{15} \mathrm{~N}\left({ }^{0}{ }_{00}\right)=\left[\frac{\left({ }^{15} \mathrm{~N} /{ }^{14} \mathrm{~N}\right)_{\text {sample }}}{\left({ }^{15} \mathrm{~N} /{ }^{14} \mathrm{~N}\right)_{\text {standard }}}-1\right] \times 1000
$$

The standard for nitrogen is atmospheric air. 


\section{Stable Carbon Isotopes}

Stable carbon isotopes have been used to determine where an animal feeds in a rainforest ecosystem (Ambrose \& DeNiro 1986). Several researchers have noted the

presence of a vertical cline (the "canopy effect") in the change in ${ }^{13} \mathrm{C}$ in leaves from the forest floor to the top of the canopy in these ecosystems (Vogel 1978; Medina \& Minchin 1980; Cormie \& Schwarcz 1994, 1996). Thus, in a rainforest ecosystem, ${ }^{13} \mathrm{C}$ is a useful indicator of habitat use. Carbon isotope composition is:

$$
\delta^{13} \mathrm{C}\left({ }^{0}{ }_{00}\right)=\left[\frac{\left({ }^{13} \mathrm{C} /{ }^{12} \mathrm{C}\right)_{\text {sample }}}{\left({ }^{13} \mathrm{C} /{ }^{12} \mathrm{C}\right)_{\text {standard }}}-1\right] \times 1000
$$

The standard for ${ }^{12} \mathrm{C}$ is the Peedee belemnite marine limestone.

\section{HYPOTHESES}

Previous research has suggested that habitat modification due to anthropogenic disturbance is reflected biosynthetically in the stable isotope composition key plant and animal species in the Ranomafana National Park ecosystem. Specifically, isotope composition of hair from lemurs inhabiting the disturbed forest (Talatekely) in RNP is depleted in stable nitrogen $\left({ }^{15} \mathrm{~N}\right)$; stable carbon $\left({ }^{13} \mathrm{C}\right)$, however, does not show any biologically significant difference between disturbed versus undisturbed forests (Vatoharanana and Valohoaka) (McGee \& Wright 2001; McGee \& Vaughn 2003, 2006, 2007; McGee et al. 2007, 2008).

The purpose of this research is to determine how habitat disturbance affects nutrient cycling and energy flow through this rainforest ecosystem as it applies to the host-parasite relationship between the ectoparasitic fly, A. crassipes (Diptera: 
Hippoboscidae) and its host, the Milne Edwards' sifika, Propithecus edwardsi (Primates: Indriidae). For stable nitrogen, the null hypothesis is that there is no difference in stable nitrogen in A. crassipes inhabiting disturbed versus undisturbed forests in RNP. The alternate hypothesis is that stable nitrogen in A. crassipes inhabiting disturbed forests will show nitrogen depletion. For stable carbon, the null hypothesis is that the patterns observed in carbon isotope composition in A. crassipes will be comparable to those seen in P. edwardsi, i.e. there will be no biologically meaningful association between carbon isotope composition and forest disturbance. The alternate hypothesis is that there will be a biologically meaningful association between carbon isotope composition and forest disturbance.

\section{MATERIALS AND METHODS}

Lemurs were darted using the Pneu-Dart system (Pneu-Dart, Inc., HC 31, Williamsport, PA). This system uses non-barbed, disposable darts with a 3/8-in needle delivered from either a blowgun or from a $\mathrm{CO}_{2}$ powered rifle. Chemical immobilization of darted lemurs is accomplished by using Telazol, a nonnarcotic anesthetic at the standard dosage of $25 \mathrm{mg} / \mathrm{kg}$ of body weight (Schoeninger et al. 1997). All protocols used in animal darting are approved by the Institutional Animal Care and Use Committee (IACUC \# 728) at San Jose State University.

After darting, the injected lemurs usually fall from the trees within 2-10 minutes and are caught using 6-ft by $10-\mathrm{ft}$ nylon nets by Malagasy darting teams. These teams carry the animals to the field research station where hair sampling and ectoparasite collection takes place. While the lemurs are anesthetized, a small amount of hair is taken 
at the base of the neck and tail for isotope analysis of the hair. The lemurs are then “combed" for ectoparasites using a cat flea comb (PETCO SKU 320951) and forceps. Table 4 shows the number of flies collected from each locality in RNP.

Table 4: Count of $A$. crassipes by Locality

\begin{tabular}{lcc}
\hline Location & $\begin{array}{c}\text { Number of flies } \\
\text { from P. edwardsi }\end{array}$ & $\begin{array}{c}\text { Average number of } \\
\text { flies per individual }\end{array}$ \\
\hline Talatakely (disturbed) & 38 & 3.8 \\
Vatoharanana (undisturbed) & 45 & 4.09 \\
Valohoaka (undisturbed) & 4 & 2 \\
\hline
\end{tabular}

\section{Specimen Preparation - Hair}

Hair specimens were washed in a 1:1 chloroform/methanol solution and then air dried. The resulting product is weighed in an analytical balance and 2-3 $\mathrm{mg}$ is placed in Sn foil boats (Costech, $5 \times 9 \mathrm{ml}$ tin capsules). Samples were shipped to the Stable Isotope Ratio Facility for Environmental Research (SIRFER) at the University of Utah for analysis on a dual inlet Finnigan MAT model 252 Isotope Ratio Mass Spectrometer (IRMS) coupled with a Carlo-Erba EA operating at continuous flow (analytical precision was $\pm 0.15 \%$.

\section{Specimen Preparation - Parasites}

Fly specimens, after being stored in $80 \% \mathrm{ETOH}$, were air dried and submerged in liquid nitrogen. Specimens were then weighed and, if less than $2 \mathrm{mg}$, placed in Sn foil boats (Costech, $5 \times 9 \mathrm{ml}$ tin capsules). Specimens over $2 \mathrm{mg}$ were cut and a $2 \mathrm{mg}$ sample 
was placed in the Sn capsule. Sample were shipped to the Stable Isotope Ratio Facility for Environmental Research (SIRFER) at the University of Utah for analysis on a dual inlet Finnigan MAT model 252 Isotope Ratio Mass Spectrometer (IRMS) coupled with a Carlo-Erba EA operating at continuous flow (analytical precision was $\pm 0.15 \%$.

\section{RESULTS}

Results from the study on lemur isotope composition (McGee \& Wright 2001; McGee \& Vaughn 2003, 2006, 2007; McGee et al. 2007, 2008) show discrete differences in the ratios of ${ }^{13} \mathrm{C}$ and ${ }^{15} \mathrm{~N}$ in $P$. edwardsi from disturbed versus undisturbed habitats. The stable carbon values range from approximately $-23 \%$ to $-24 \%_{0}$ in Talatekely and from $-23.3 \%$ to $-22.5 \%$ in Vatoharanana and Valohoaka. The stable nitrogen values range from approximately $1.5 \%$ to $2.9 \%$ in Talatekely and from $2.9 \%$ o to $4 \%$ in Vatoharanana and Valohoaka. Figure 5 shows stable nitrogen plotted against stable carbon for P. edwardsi in disturbed versus undisturbed forests.

Although differences are seen in both stable carbon and nitrogen, only nitrogen could be considered biologically significant. Figure 5 shows a maximum of $4 \%$ for lemurs inhabiting undisturbed sites and a minimum of $1.5 \%$ for lemurs inhabiting disturbed sites. As noted previously, each trophic level is represented by a 3\%o difference in stable nitrogen. Therefore, a difference of $2.5 \%$ in the same species of lemur inhabiting forests that are comparable except in degree of disturbance is truly exceptional. These differences are verified statistically (Single Factor ANOVA, $p<.001$; $\mathrm{df}=53$ ). The range of values in lemurs for stable carbon between disturbed and undisturbed forest, 


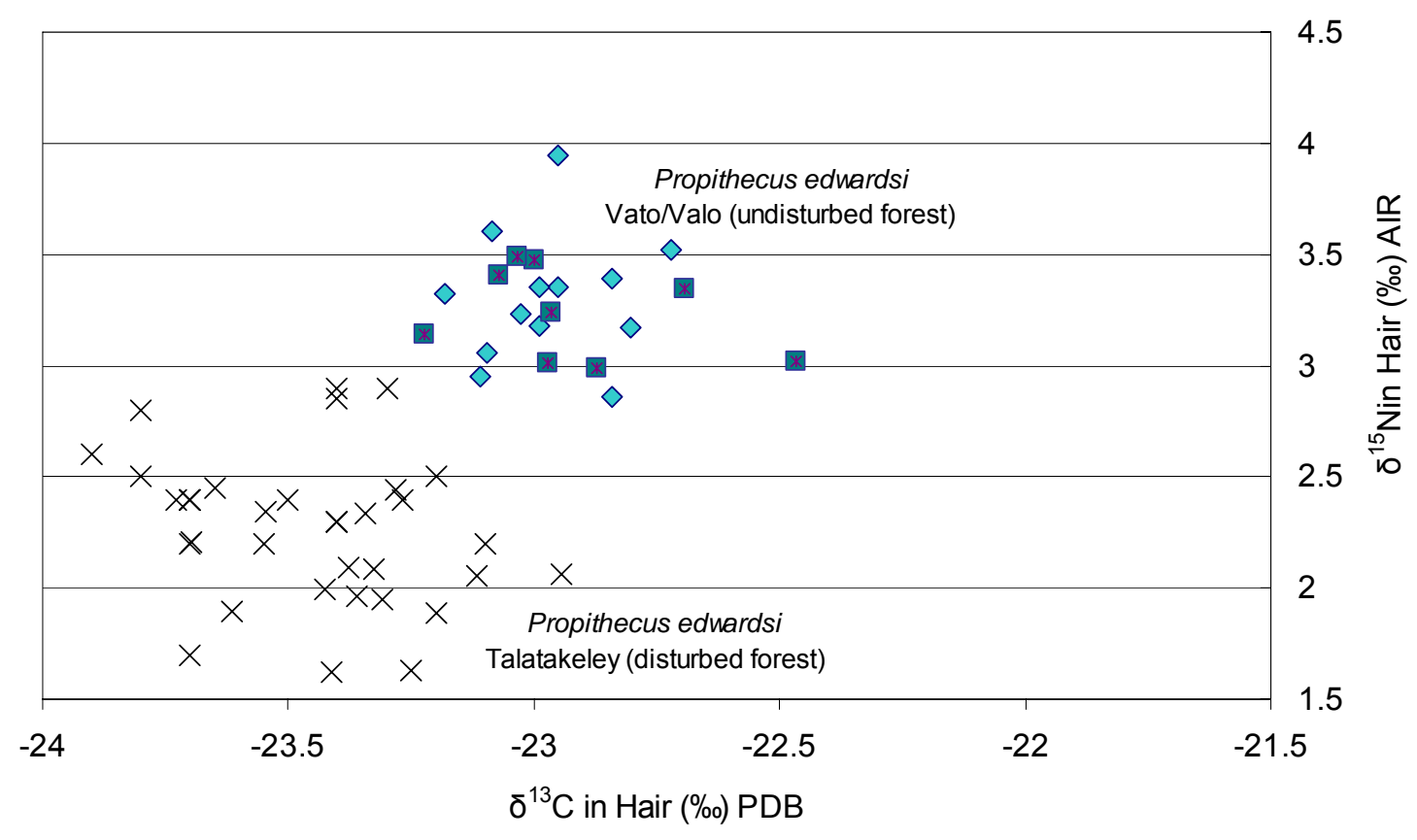

Figure 5: Biogeochemical Profile of Propithecus edwardsi 
on the other hand is at most $1.5 \%$ and while is statistically significant (Single Factor ANOVA, $p<.001 ; \mathrm{df}=53$ ) is unlikely to be a biologically significant difference.

Results from this study on isotope composition in A. crassipes also show discrete differences in the ratios of ${ }^{13} \mathrm{C}$ and ${ }^{15} \mathrm{~N}$ from disturbed versus undisturbed forests. The stable carbon values range from $-23.9 \%$ o to $-25.7 \%$ in Talatekely and from $-24.2 \%$ to $24.8 \%$ in Vatoharanana. The stable nitrogen values range $7.4 \%$ to $8.5 \%$ in Talatekely and from $8.6 \%$ to $9.7 \%$ in Vatoharanana (Table 5).

Table 5: Stable isotope values for $A$. crassipes

\begin{tabular}{|c|c|c|c|c|c|c|c|}
\hline & \multirow[b]{2}{*}{$\mathrm{n}$} & \multicolumn{3}{|c|}{$\delta^{15} \mathrm{~N}(\mathrm{AIR})(\%)$} & \multicolumn{3}{|c|}{$\delta{ }^{13} \mathrm{C}(\mathrm{PDB})(\%$ ) } \\
\hline & & $\mathrm{x}$ & $\mathrm{SD}$ & Range & $\mathrm{x}$ & $\mathrm{SD}$ & Range \\
\hline $\begin{array}{l}\text { A. crassipes } \\
\text { Talatekely }\end{array}$ & 5 & 8.0 & 0.43 & $7.4,8.5$ & 24.8 & 0.6 & $-25.7,-23.9$ \\
\hline $\begin{array}{l}\text { A. crassipes } \\
\text { Vataharanana }\end{array}$ & 7 & 9.0 & 0.36 & $8.6,9.7$ & 24.5 & 0.2 & $-24.8,-24.2$ \\
\hline
\end{tabular}

Figure 6 shows stable nitrogen plotted against stable carbon for A. crassipes in disturbed versus undisturbed forests. Stable carbon and nitrogen isotopes for the disturbed forest of Talatekely and the undisturbed forest of Vatoharanana form distinct clusters. Stable nitrogen values for A. crassipes feeding on lemurs from disturbed versus undisturbed forests are significantly different (Single Factor ANOVA, $p=.001$, df $=11$ ).

There is no significant difference in the stable carbon isotope composition of $A$. crassipes feeding on lemurs in disturbed versus undisturbed forests (Single Factor ANOVA, $p=.31, \mathrm{df}=11)$. The values of ${ }^{13} \mathrm{C}$ of $A$. crassipes is between -24 and -25.5 , 


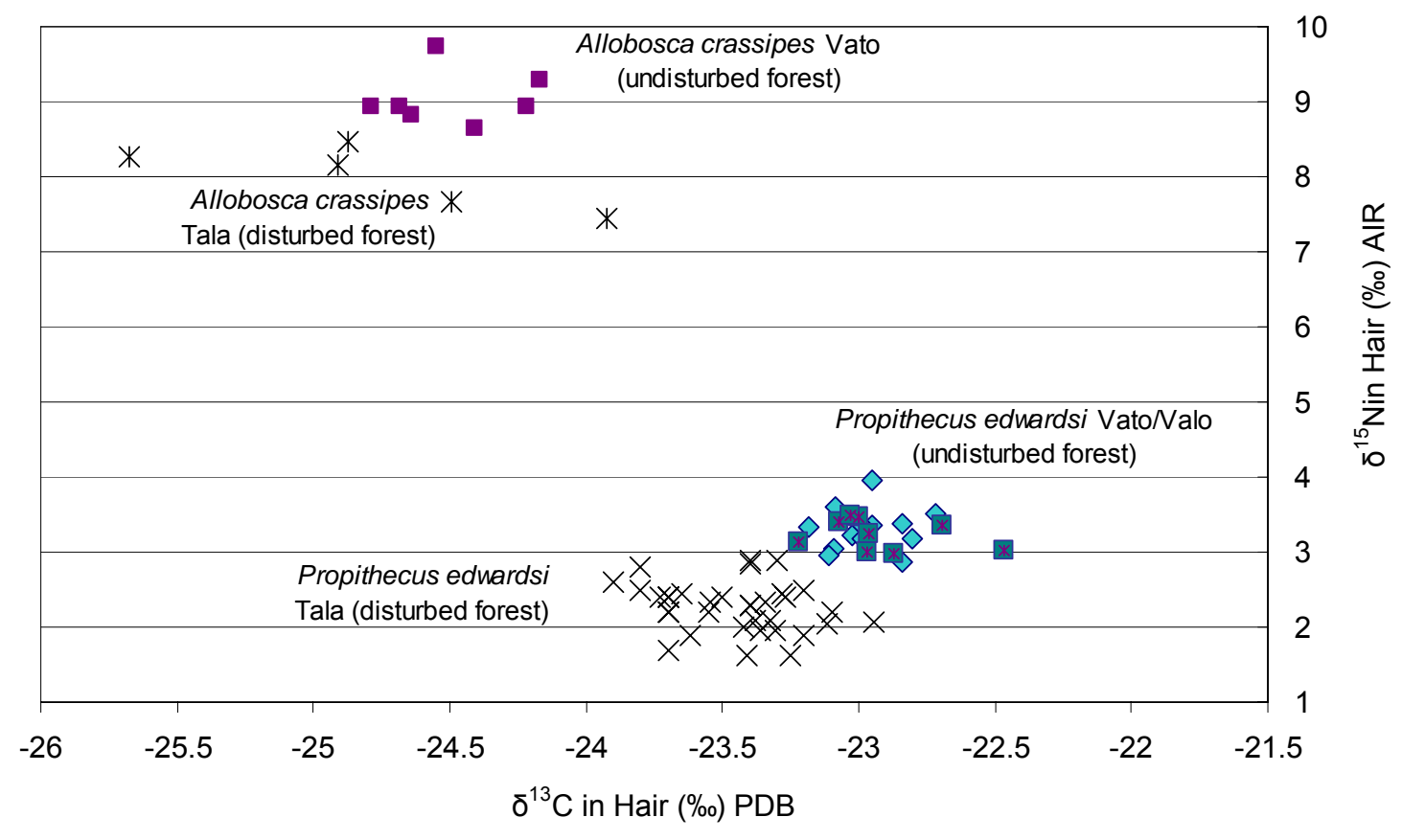

Figure 6: Biogeochemical Profile of Allobosca crassipes with Propithecus edwardsi 
and spans both Talatekely and Vatoharanana. The range of ${ }^{13} \mathrm{C}$ in lemurs is comparable (1.5\%), however, within this range the lemurs from Talatekely are more carbon depleted than the lemurs from Vatoharanana so the differences are statistically different. As noted previously, a difference of $1.5 \%$ in stable carbon (which in $\mathrm{C}_{3}$ plants range from -31 to 33 ) is not likely to be biologically significant.

\section{CONCLUSION}

I analyzed flies collected from P. edwardsi in Talatekeley, Vatoharanana and Valohoaka to compare their isotope ratios with those of the lemurs. The isotope ratios observed in the flies collected from $P$. edwardsi track the depletion of stable nitrogen observed in lemur hair from previous studies at these sites. The data presented here support the hypothesis that the stable nitrogen composition of A. crassipes collected from lemurs inhabiting disturbed forests is depleted compared to flies collected from lemurs in undisturbed forests. Further, the patterns in stable carbon isotopes of $A$. crassipes between disturbed and undisturbed habitats are comparable to those seen in P. edwardsi, i.e., there is no association between stable carbon isotopes and habitat disturbance.

On average, the stable nitrogen isotope composition of $A$. crassipes is one trophic level greater than that of $P$. edwardsi, and a distinct difference is seen in the flies and lemurs inhabiting disturbed and undisturbed forests. These data further validate stable isotope ecology as a means of identifying habitat disturbance at the level of ecosystem in rain forests. 


\section{CHAPTER V: Conclusions}

The limited sample size and the degraded condition of the specimens of Allobosca crassipes examined by previous researchers historically have made the identification and the differentiation of species and sex of this fly problematic. These earlier descriptions of key morphological features such as the abdominal and wing characteristics are at best, inconsistent. The collection and curation of the 101 specimens collected in 2000-2001 provide the most comprehensive assemblage of $A$. crassipes yet established for taxonomic use in the description of this species. The observations recorded from my examination of this modern collection provide continuity and clarity in the use of these key characters used in the identification of $A$. crassipes. The 87 specimens housed in the J. Gordon Edwards Museum at San Jose State University represent the largest collection known, for use in study of the taxonomy and systematics of A. crassipes.

Host specificity in the family Hippoboscidae has been described as moderately high, with flies being found on unrelated host species in similar habitats Maa (1963). The new host association of A. crassipes with the Black-and-White Ruffed lemur, Varecia variegata variegata and confirmed host association with the Milne-Edwards sifaka, Propithecus edwardsi is further evidence that supports the pleioxeny of $A$. crassipes, first suggested by Bequaert (1953). The postulated wing-shedding of the louse fly render it flightless, but these non-vagile flies still have opportunity for host acquisition if the lemurs light on the ground. The geophagous behavior of $V$. variegata variegata and P. edwardsi present questing flies increased opportunities the parasite to acquire a host and expand its host associations. Clearly, host specificity in A. crassipes is higher 
than previously thought, and the new association with $V$. variegata variegata invites further investigation into the ecology of this parasite and its hosts.

Regarding ecosystem level effects of habitat disturbance, the depletion of stable nitrogen of $A$. crassipes in disturbed habitats tracks with the depletion seen in the lemurs feeding in disturbed habitats (McGee \& Vaughn 2003). The predicted enrichment of stable nitrogen in the flies collected from lemurs in undisturbed forests is further evidence of the use of stable isotope analysis as a tool in quantifying trophic changes in the rainforest ecosystem due to anthropogenic activity. 


\section{LITERATURE CITED}

Ambrose, S. H. \& DeNiro, M. J. 1986. The isotopic ecology of East African mammals. Oecologia 69:395-406.

Ambrose, S..H \& DeNiro, M. 1986. Reconstruction of African human diet using bone collagen carbon and nitrogen isotope ratios. Nature 319:321-324.

Arrigo-Nelson, S. J. 2006. The impact of habitat disturbance on the feeding ecology of the Milne-Edwards' sifaka (Propithecus edwardsi) in Ranomafana National Park, Madagascar. Ph.D. dissertation, SUNY-Stony Brook, Stony Brook, NY.

Arrigo-Nelson, S. J., Baden, A. L., \& Wright, P. C. In press. Testing hypotheses for geophagy in a Malagasy rainforest lemur. Biotropica.

Balgooyen, T. G., Hallmann, B. \& Vaughn, S.E. 1999. A new host record of Ornithophila gestroi (Diptera: Hippoboscidae) on the lesser kestrel (Falco naumanni Fleischer) in Galaxidi, Greece. Pan Pacific Entomologist 75:60.

Balko, E. A. 1998. A behaviorally plastic response to forest composition and logging disturbance in Varicia variegata variegata in Ranomafana National Park. Ph.D. dissertation, Syracuse, State University of New York.

Balko, E. A. \& Underwood, H. B. 2005. Effects of forest structure and composition on food availability for Varecia variegata at Ranomafana National Park, Madagascar. American Journal of Primatology 66:45-70.

Bennett, E. T. 1832. Characters of a new genus of Lemuridae (Propithecus), presented by Mr. Telfair. Proceedings of the Committee of Science and Correspondence of the Zoological Society of London Part II:20-46.

Bequaert, J. C. 1952. The Hippoboscidae or louse-flies (Diptera) of mammals and birds. Part I. Structure, physiology and natural history. Entomologica Americana 32:1-209.

Bequaert, J. C. 1953. The Hippoboscidae or louse-flies (Diptera) of mammals and birds. Part I. Structure, physiology and natural history. Entomologica Americana 33:211-442.

Burk, R. L. \& Stuiver. M. 1981. Oxygen isotope ratios in trees reflect mean annual temperature and humidity. Science 211:1417-1419.

Cormie, A. B. \& Schwarcz, H. P. 1994. Climatic influence on bone nitrogen and carbon isotopes in North American white-tailed deer. Paleogeography, Paleoclimatology, Paleoecology 107:227-241. 
Cormie, A. B. \& Schwarcz, H. P. 1996. Effects of climate on deer bone $\delta^{15} \mathrm{~N}$ and $\delta^{13} \mathrm{C}$ : Lack of precipitation effects on $\delta^{15} \mathrm{~N}$ for animals consuming low amounts of $\mathrm{C} 4$ plants. Geochimica et Cosmochimica Acta 60:4161-4166.

DeNiro, M J. \& Epstein, S. 1981. Influence of diet on the distribution of nitrogen isotopes in animals. Geochimica et Cosmochimica Acta 45:341-351.

Dew, J. L. \& Wright, P. C. 1998. Frugivory and seed dispersal by four species of primates in Madagascar's eastern rain forest. Biotropica 30:425-437.

Dick, C. 2006. Checklist of World Hippoboscidae (Diptera: Hippoboscidae) http://fm1.fieldmuseum.org/aa/Files/cdick/Hippoboscidae_Checklist_20dec06.pdf.

Evenhuis, N. L. 2009. Personal Communication on June 02, 2009. Bishop Museum, Hawaii.

Ferris, G. F. \& Cole, F. R. 1922. A contribution to the knowledge of the Hippoboscidae (Diptera Pupipara). Parasitology 14:178-205.

Garbutt, N. 1999. Mammals of Madagascar. Yale University Press, New Haven. 1-320.

Geoffroy Saint-Hilaire, I. 1850. Note sur plusieurs espèces nouvelles de mammifères de l'ordre des primates. Les Comptes Rendus de l'Académie des Sciences 31:873-876.

Geoffroy Saint-Hilaire, I. 1851. Catalogue méthodique de la collection des mammifères de la collection des oiseaux et des collection annexes. Muséum National d'Histoire Naturelle, Gide et Baudry, Paris, 1-96 pp.

Goodman, S. M. \& Benstead, J. P. 2003. The Natural History of Madagascar. Chicago: University of Chicago Press. 1-1709.

Grandidier, A. 1871. Observations sur les Propithèques de Madagascar. Les Comptes Rendus de l'Académie des Sciences 72:231-232.

Groves, C. P. \& Helgen, K. M. 2007. Craniodental characters in the taxonomy of Propithecus. International Journal of Primatology 28:1363-1383.

Kerr, R. 1792. The Animal Kingdom, or Zoological System, of the Celebrated Sir Charles Linnceus: Class I. Mammalia. J. Murray and R. Faulder, London, pp. 1-664.

Koch, P. L., Fogel, M. L. \& Tuross, N. 1994. Tracing the diets of fossil animals using stable isotopes. In K. Lajtha \& R.H. Michener (eds), Stable Isotopes in Ecology and Environmental Science 63-92. Oxford: Blackwell Scientific. 
Krause, D. W.2003. Late Cretaceous vertebrates of Madagascar: a window into Gondwandan biogeography at the end of the age of dinosaurs. In S. M. Goodman \& J. P. Benstead (eds), The Natural History of Madagascar 40-47. Chicago: University of Chicago Press.

Lavauden, L. 1931. Un nouveau Propithèque de Madagascar (Propithecus perrieri, sp. nov.). Comptes Rendus Hebdomadaires des Seances de l'Academie des Sciences 193: 7779.

Linnaeus, C. 1766. Systema naturae per regna tria naturae. Edition 12, Volume 1. Impensis Laurentii Salvii (Holmiae). Stockholm, Sweden, p. 1-532.

Linnaeus, C. (revised by J. F. Gmelin). 1788. Systema naturae per regna tria naturae. Edition 13, Volume 1. G. E. Beir, Lipsiae, p. 1-232.

Maa, T. C. 1963. Genera and species of Hippoboscidae: types, synonymy, habitats and natural groupings. Pacific Insects Monograph 6:1-186.

Maa, T. C. 1969. A revised checklist and concise host index of Hippoboscidae (Diptera). Pacific Insects Monograph 20:261-299.

Macko, S. A. \& Engel, M. H. 1991. Assessment of indigeneity in fossil organic matter, amino acids and stable isotopes. Philosophical Transactions of the Royal Society of London B 333:367-374.

Mayor, M. I., Sommer, J. A., Houck, M. L., Zaonarivelo, J. R., Wright, P., Ingram, C., Engel, S. R. \& Louis, Jr., E. E. 2004. Specific Status of Propithecus spp. International Journal of Primatology 25:875-900.

McGee, E. M. \& Wright, P. C. 2001. Stable isotope composition in Propithecus diadema edwardsi from Ranomafana National Park, Madagascar. American Journal of Physical Anthropology Supplement 32:106.

McGee, E. M. \& Vaughn, S. E. 2003. Variations in stable isotope composition in Propithecus diadema edwardsi from disturbed and undisturbed rainforest habitats in Ranomafana National Park, Madagascar. American Journal of Physical Anthropology Supplement 36:149-150.

McGee, E. M. \& Vaughn, S. E. 2006. Stable isotope analysis: a technique for evaluating ecological change in disturbed habitats. International Journal of Primatology (Supplement 1) 27:499. 
McGee, E. M. \& Vaughn, S. E. 2007. Biogeochemical effects of anthropogenic disturbance on Propithecus edwardsi (Primates: Indriidae) from Ranomafana National Park in southeastern Madagascar. American Journal of Physical Anthropology Supplement 44:168.

McGee, E. M., Vaughn, S. E. \& Wright, P. C. 2002. Stable isotope composition in Propithecus diadema edwardsi from Talatakely and Vatoharanana in Ranomafana National Park, Madagascar. American Journal of Physical Anthropology Supplement 34:110.

McGee, E. M., Vaughn, S. E. \& Lundberg, J. S. 2008. Biogeochemical effects of anthropogenic disturbance across time and habitat in Madagascar. International Journal of Primatology 96:447.

Medina, E. \& Minchin, P. 1980. Stratification of $\delta^{13} \mathrm{C}$ values of leaves in Amazonian rain forests. Oecologia 45:377-378.

Minagowa, M. \& Wada, E. 1984. Stepwise enrichment of ${ }^{15} \mathrm{~N}$ along food chains; further evidence and the relation between ${ }^{15} \mathrm{~N}$ and animal age. Geochimica et Cosmochimica Acta 481:1135-1140.

Myers, N., Mittermeier, R. A., Mittermeier, C. G., da Fonseca, G. A. B., Kent, J. 2000. Biodiversity hotspots for conservation priorities. Nature 403:853-858.

Overdorff, D. J., Erhart, E. M., \& Mutschler, T. 2005. Does female dominance facilitate feeding priority in black-and-white ruffed lemurs (Varecia variegata) in southeastern Madagascar? American Journal of Primatology 66:7-22.

Petter, J.-J., Albignac, R. \& Rumpler, Y. 1977. Mammifères Lémuriens (Primates Prosimiens). Faune de Madagascar. No. 44. ORSTOM-CNRS, Paris, pp. 1-513.

Pochron, S. T., Tucker, W. T. \& Wright, P. C. 2004. Demography, Life History, and Social Structure in Propithecus diadema edwardsi From 1986-2000 in Ranomafana National Park, Madagascar. American Journal of Physical Anthropology 125:61-72.

Rabinowitz, P. D., Coffin, M. C., \& Falvey, D. 1983. The separation of Madagascar and Africa. Science 220:67-69.

Schoeninger, M. J., Iwaniec, U. T. \& Glander, K. E. 1997. Stable isotope ratios indicate diet and habitat use in New World monkeys. American Journal of Physical Anthropology 103:69-83.

Schoeninger, M. J., Iwaniec, U. T. \& Glander, K. E. 1998. Ecological attributes recorded in stable isotope ratios in arborial prosimian hair. Oecologia 113:222-230. 
Speiser, P. 1899. Eine neue, auf Halbaffen lebende Hippobosciden-Art (Dipt.). Wiener Entomologische Zeitung 18:197-202.

Tattersall, I. 1982. The Primates of Madagascar. Columbia University Press, New York, pp. 1-382.

Tattersall, I. 1986. Notes on the distribution and taxonomic status of some subspecies of Propithecus in Madagascar. Folia primatologica 46:51-63.

Theodor, O. \& Oldroyd, H. 1965. On a new genus and species of Hippoboscidae (Diptera) and its relationship to Allobosca crassipes Speiser. Proceedings of the Royal Entomological Society, London (B) 34 (7-8):91-97.

Vogel, J. C. 1978. Recycling of carbon in a forest environment. Ecologica Plantarum 13:89-94.

Vogel, J. C. \& van der Merwe, N. J. 1977. Isotopic evidence for early maize cultivation in New York State. American Antiquity 42:238-242.

White, F. J. 1989. Diet, ranging behavior and social organization of the black and white ruffed lemur, Varecia variegata variegata in southeastern Madagascar. American Journal of Physical Anthropology 78:323.

White, F. J., Overdorff, D. J., Balko, E. A. \& Wright, P. C. 1995. Distribution of ruffed lemurs(Varecia variegata variegata) in Ranomafana National Park. Folia Primatologica 64:124-131.

Wright, P. C. 1992. Primate ecology, rainforest conservation, and economic development: building a national park in Madagascar. Evolutionary Anthropology 1:2533.

Wright, P. C. 1997. The future of biodiversity in Madagascar - a view from Ranomafana National Park. In S. Goodman and B. Patterson (eds), Natural Change and Human Impact on Madagascar 381-405. Smithsonian Institution Press.

Wright, P. C., Arrigo-Nelson, S. J., Hogg, K. L., Bannon, B., Morelli, T. L., Wyatt, J., Harivelo, A. L. \& Ratelolahy, F. 2009. Habitat disturbance and seasonal fluctuation of lemur parasites in the rain forest of Ranomafana National Park, Madagascar, pp. 311-330. In: M. Huffman \& C. Chapman (Eds.). Primate Parasite Ecology: The Dynamics and Study of Host-Parasite Relationships. Cambridge University Press, Cambridge.

Yapp, C. J. \& Epstein, S. 1982. Climatic significance of the hydrogen isotope ratios in tree cellulose. Nature 297:636-639. 